\title{
Neutrino transport in black hole-neutron star binaries: Neutrino emission and dynamical mass ejection
}

\author{
Koutarou Kyutoku ${ }^{1,2,3,4}$, Kenta Kiuchi ${ }^{4}$, Yuichiro Sekiguchi ${ }^{5,4}$, Masaru Shibata ${ }^{6,4}$, and Keisuke Taniguchi ${ }^{7}$ \\ ${ }^{1}$ Theory Center, Institute of Particle and Nuclear Studies, KEK, Tsukuba 305-0801, Japan \\ ${ }^{2}$ Department of Particle and Nuclear Physics, the Graduate \\ University for Advanced Studies (Sokendai), Tsukuba 305-0801, Japan \\ ${ }^{3}$ Interdisciplinary Theoretical Science (iTHES) Research Group, RIKEN, Wako, Saitama 351-0198, Japan \\ ${ }^{4}$ Center for Gravitational Physics, Yukawa Institute for Theoretical Physics, Kyoto University, Kyoto 606-8502, Japan \\ ${ }^{5}$ Department of Physics, Toho University, Funabashi, Chiba 274-8510, Japan \\ ${ }^{6}$ Max Planck Institute for Gravitational Physics (Albert Einstein Institute), \\ Am Mühlenberg 1, Postdam-Golm 14476, Germany \\ ${ }^{7}$ Department of Physics, University of the Ryukyus, Nishihara, Okinawa 903-0213, Japan
}

(Dated: January 23, 2018)

\begin{abstract}
We study the merger of black hole-neutron star binaries by fully general-relativistic neutrinoradiation-hydrodynamics simulations throughout the coalescence, particularly focusing on the role of neutrino irradiation in dynamical mass ejection. Neutrino transport is incorporated by an approximate transfer scheme based on the truncated moment formalism. While we fix the mass ratio of the black hole to the neutron star to be 4 and the dimensionless spin parameter of the black hole to be 0.75 , the equations of state for finite-temperature neutron-star matter are varied. The hot accretion disk formed after tidal disruption of the neutron star emits a copious amount of neutrinos with the peak total luminosity $\sim 1-3 \times 10^{53} \mathrm{erg} \mathrm{s}^{-1}$ via thermal pair production and subsequent electron/positron captures on free nucleons. Nevertheless, the neutrino irradiation does not modify significantly the electron fraction of the dynamical ejecta from the neutrinoless $\beta$-equilibrium value at zero temperature of initial neutron stars. The mass of the wind component driven by neutrinos from the remnant disk is negligible compared to the very neutron-rich dynamical component, throughout our simulations performed until a few tens milliseconds after the onset of merger, for the models considered in this study. These facts suggest that the ejecta from black hole-neutron star binaries are very neutron rich and are expected to accommodate strong $r$-process nucleosynthesis, unless magnetic or viscous processes contribute substantially to the mass ejection from the disk. We also find that the peak neutrino luminosity does not necessarily increase as the disk mass increases, because tidal disruption of a compact neutron star can result in a remnant disk with a small mass but high temperature.
\end{abstract}

PACS numbers: 04.25.D-, 04.30.-w, 04.40.Dg

\section{INTRODUCTION}

Mass ejection from the merger of black hole-neutron star binaries has been studied vigorously in recent years 11-14, as well as that from binary-neutron-star mergers [15 21. Neutron-rich material ejected from black hole-neutron star binaries can accommodate $r$-process nucleosynthesis, which produces about a half of neutronrich nuclei heavier than the iron in the Universe [22]. This nucleosynthesis is important not only for cosmic chemical evolution but also for electromagnetic transient radiation, which serves as electromagnetic counterparts to gravitational-wave sources [7. In particular, the decay of unstable $r$-process elements heats up the ejecta, and associated quasithermal radiation, so-called macronova/kilonova, will be observed in infrared-optical bands on the time scale of a week 23 27. While five gravitational-wave events from binary black holes were detected successfully $28+32$, the nature of many weak signal candidates including LVT151012 [33] remains elusive. If we could simultaneously detect accompanying electromagnetic counterparts, gravitational waves from the merger of black hole-neutron star binaries may be securely identified as astrophysical.

The mass ejection and electromagnetic counterparts are also important to reveal the nature of shorthard gamma-ray bursts (see Refs. [34 36] for reviews). A near-infrared transient detected in the afterglow of GRB 130603B is consistent with models of the macronova/kilonova 37-39, supporting the binarymerger hypothesis of short-hard gamma-ray bursts. While this transient is detected only in a single epoch, future detailed observations of the macronovae/kilonovae will give us valuable information of their progenitors as well as mass ejection mechanisms, and appropriate interpretation requires firm understanding of the ejecta properties such as the mass and velocity. At the same time, if the material is ejected along the polar axis of the remnant black hole and surrounding accretion disk, the ejecta could also affect the propagation of a possible gamma-ray burst jet via baryon loading and hydrodynamical collimation 40 44. Thus, the geometry of mass ejection is also a subject of investigation for accurately understanding the short-hard gamma-ray burst.

Indeed, the first multimessenger detections of a binary-neutron-star merger event, 
GW170817/GRB 170817A/AT 2017gfo, was announced during the review process of this article 45 47. The outlook envisioned above is now broadly confirmed for binary neutron stars except that GRB 170817A is not likely to be associated with an ultrarelativistic jet [48, 49. Still, the mechanism of mass ejection needs clarification to understand observed electromagnetic emission. In anticipation of forthcoming detections of black hole-neutron star binaries, detailed studies of their mass ejection have also become increasingly important.

General relativity, radiation hydrodynamics, and neutrino transport are all essential to study quantitatively the mass ejection from compact binary mergers and subsequent nucleosynthesis. Recent neutrino-radiationhydrodynamics simulations in full general relativity have shown that the electron fraction $Y_{e}$ of dynamical ejecta from binary neutron stars can have a broad distribution with $Y_{e} \sim 0.05-0.5$ due to the strong shock heating and neutrino irradiation [17, 19, 20, 50. This should be contrasted with Newtonian or approximately generalrelativistic simulations, by which the distribution is always predicted to be concentrated in $Y_{e} \lesssim 0.1$, characteristic of neutron stars in neutrinoless $\beta$-equilibrium at zero temperature [51 53]. Broad distribution of $Y_{e}$ is a key to reproduce the solar pattern of $r$-process abundances in a single event [50, 54, 55] and may be advantageous to explain the universality of the abundance pattern observed in $r$-process-enhanced metal-poor stars [56, 57]. Furthermore, neutrino irradiation can also trigger neutrinodriven mass ejection, or neutrino-driven wind, in the postmerger phase [58 61. (see also Refs. [13, 17, 19[21, 62, 63]). Even if the dynamical ejecta do not have sufficiently broad distribution of $Y_{e}$, such disk winds could play a crucial role in reproducing the solar pattern [13, 64.

In this paper, we investigate the role of neutrinomatter interaction during the merger process of black hole-neutron star binaries by numerical-relativity simulations. While fully general-relativistic simulations of the mass ejection from black hole-neutron star binaries have been extensively performed adopting nucleartheory-based equations of state by various researchers [7-12, 14, the neutrino transport has been limited to the cooling [8, 9, 14] except for the study focusing only on the postmerger evolution 65. Our simulations incorporate both neutrino cooling and heating in an approximate but self-consistent manner throughout the inspiralmerger-postmerger phases in full general relativity. Various nuclear-theory-based finite-temperature equations of state are adopted to explore dependence of the merger outcome on underlying microphysics. In this study, we focus particularly on the neutrino emission and mass ejection from black hole-neutron star binary mergers.

This paper is organized as follows. Section II describes our numerical scheme and adopted models. Results of neutrino-radiation-hydrodynamics simulations are presented in Sec. III and they are compared with results of simulations without neutrino absorption in Sec. IV to
TABLE I. Key ingredients of the adopted equations of state. $M_{\text {max }}$ is the maximum mass of a spherical neutron star at zero temperature. $M_{*, 1.35}, R_{1.35}, \mathcal{C}_{1.35}$, and $\Lambda_{1.35}$ are the baryon rest mass $M_{*}$, circumferential radius $R$, and compactness $\mathcal{C} \equiv$ $G M_{\mathrm{NS}} /\left(R c^{2}\right)$ with $M_{\mathrm{NS}}$ being the gravitational mass, and dimensionless tidal deformability $\Lambda$ of a $1.35 M_{\odot}$ neutron star, respectively.

\begin{tabular}{c|ccccc}
\hline Model & $M_{\max }\left[M_{\odot}\right]$ & $M_{*, 1.35}\left[M_{\odot}\right]$ & $R_{1.35}(\mathrm{~km})$ & $\mathcal{C}_{1.35}$ & $\Lambda_{1.35}$ \\
\hline \hline SFHo & 2.06 & 1.48 & 11.9 & 0.167 & 420 \\
DD2 & 2.42 & 1.47 & 13.2 & 0.151 & 854 \\
TM1 & 2.21 & 1.45 & 14.5 & 0.138 & 1428 \\
\hline
\end{tabular}

single out the effect of neutrino transport. Sections V and $\mathrm{VI}$ are devoted to discussions and a summary, respectively. The gravitational constant and speed of light are denoted by $G$ and $c$, respectively. The temperature is expressed in $\mathrm{MeV}$, implicitly multiplying the Boltzmann constant, $k_{\mathrm{B}}$. The greek indices $\alpha$ and $\beta$ denote spacetime components, and the latin index $i$ denotes space components.

\section{NUMERICAL METHOD}

\section{A. Equation of state and initial condition}

We adopt three nuclear-theory-based finitetemperature equations of state for the neutron-star matter to span a plausible range of nuclear-matter properties following our previous work on binary neutron stars [17. Specifically, the so-called SFHo [66], DD2 67, and TM1 68] equations of state are employed in this study. Our choice is also the same as that for black hole-neutron star binary mergers performed in a conformal flatness approximation of Ref. [13. The equations of state provide thermodynamic quantities such as the pressure and entropy as functions of the rest-mass density $\rho$, electron fraction $Y_{e}$, and temperature $T$. Important properties of neutron stars computed with the adopted equations of state are summarized in Table 1 (see also Ref. [17] for microphysics parameters). The radii of $1.35 M_{\odot}$ neutron stars at zero temperature are $11.9 \mathrm{~km}, 13.2 \mathrm{~km}$, and $14.5 \mathrm{~km}$ for SFHo, DD2, and TM1, respectively. While TM1 is marginally inconsistent with existing constraints 69, 70, 1 we include this model for exploring the dependence of merger outcomes on equations of state in a wide range. All the equations of state can support observed $2 M_{\odot}$ neutron stars [71, 72].

Initial data of black hole-neutron star binaries are given by quasiequilibrium states computed in the puncture framework. Numerical computations are performed using a multidomain spectral method library, LORENE[2

\footnotetext{
1 TM1 is not favored from the observation of GW170817 [45] either, which was recently.

2 http://www.lorene.obspm.fr/
} 
and the details are described in Refs. [3, 73. Neutrinoless $\beta$-equilibrium states at $0.1 \mathrm{MeV}$ are assumed for the initial neutron stars. The electron fraction typically takes values of $0.05-0.1$ for the inner crust and the outer core, from which dynamical ejecta arise [11].

In this work, we fix all the parameters except for the neutron-star equations of state for simplicity. The masses of black holes and neutron stars are chosen to be $M_{\mathrm{BH}}=5.4 M_{\odot}$ and $M_{\mathrm{NS}}=1.35 M_{\odot}$, respectively. Thus, the mass ratio $Q \equiv M_{\mathrm{BH}} / M_{\mathrm{NS}}$ is 4 . This relatively small value is chosen so that the neutron star can be disrupted before the plunge in the presence of a moderately large spin of the black hole [3, 11], while the mass is kept astrophysically realistic [74]. The dimensionless spin parameter of the black hole is chosen to be $\chi \equiv c J_{\mathrm{BH}} /\left(G M_{\mathrm{BH}}^{2}\right)=0.75$ with $J_{\mathrm{BH}}$ being the angular momentum of the black hole, and the orientation is set to be parallel to the orbital angular momentum of the binary. The initial orbital angular velocity $\Omega$ of the binary is chosen to be $G m_{0} \Omega / c^{3}=0.056$, with which binaries spend $\sim 3-4$ orbits before tidal disruption of neutron stars.

\section{B. Radiation-hydrodynamics simulation}

Our numerical simulations are performed by a fully general-relativistic neutrino-radiation-hydrodynamics code developed in Refs. [17, 19. The Einstein evolution equations are solved in the Baumgarte-Shapiro-ShibataNakamura formalism [75, 76] employing a moving puncture gauge condition [77 79]. Radiation-hydrodynamics equations for neutrinos are solved explicitly in time by an approximate neutrino-transfer scheme based on the truncated moment formalism [80, 81] with cooling source terms computed by a general-relativistic leakage scheme [82, 83, and heating sources terms due to neutrino capture processes (see also Ref. 61]). Specifically, we decompose the energy-momentum tensor and associated local conservation equations into two parts. One part denoted by $T^{\alpha \beta}$ represents the sum of the fluid and trapped neutrinos, for which the basic equation is given by

$$
\nabla_{\beta} T^{\alpha \beta}=-Q_{\text {cool }}^{\alpha}+Q_{\text {heat }}^{\alpha}
$$

The other part denoted by $T_{\nu, \mathrm{S}}^{\alpha \beta}$ represents streaming neutrinos, and the basic equation is

$$
\nabla_{\beta} T_{\nu, \mathrm{S}}^{\alpha \beta}=Q_{\mathrm{cool}}^{\alpha}-Q_{\text {heat }}^{\alpha} .
$$

Here, $Q_{\text {cool }}^{\alpha}$ and $Q_{\text {heat }}^{\alpha}$ are the rates of neutrino emission (cooling) and absorption (heating), respectively. The energy-momentum tensor of the fluid and trapped neutrinos is written by the ideal-fluid form with the four velocity $u^{\alpha}$ [82, and that of streaming neutrinos is given assuming an M1 closure relation with a variable Eddington factor for handling gray regimes 81 . We also
TABLE II. Grid setup of each model. $L$ is the box size of the largest computational domain. $\Delta x$ is the grid spacing at the finest computational domain. $R_{\text {diam }} / \Delta x$ is the number of grids assigned to the semimajor diameter of the neutron star along the binary separation.

\begin{tabular}{c|ccc}
\hline Model & $L(\mathrm{~km})$ & $\Delta x(\mathrm{~m})$ & $R_{\text {diam }} / \Delta x$ \\
\hline \hline SFHo & 17600 & 250 & 70 \\
DD2 & 18040 & 270 & 75 \\
DD2-low & 18432 & 400 & 52 \\
TM1 & 19507 & 300 & 76 \\
\hline
\end{tabular}

solve the evolution of chemical composition incorporating both the neutrino emission and absorption, applying a $\beta$-equilibrium limiter on the electron fraction to avoid an unstable oscillation associated with stiff source terms 82. The floor density of artificial atmosphere is set to be $\approx 2 \times 10^{3} \mathrm{~g} \mathrm{~cm}^{-3}$ with $Y_{e}=0.47$ and $T=0.1 \mathrm{MeV}$ in this work.

The emission and absorption rates are determined by an optical-depth-weighted sum of the values for the diffusion and free-streaming limits [82. Our emission processes include electron/positron captures on free nucleons, those on heavy nuclei, pair annihilation, nucleon bremsstrahlung, and plasmon decay. Precise forms of the emission rates are provided in Ref. 84. For the absorption, we consider neutrino captures on free nucleons. The absorption rate is computed using the cross section given in Ref. 84 assuming neutrinos to obey the Fermi-Dirac distribution at the fluid temperature with chemical potential that reproduces the energy density obtained from time evolution. In fact, this assumption on the temperature is not always well motivated, and we discuss this issue in Sec. IV We collectively denote muon/tau neutrinos/antineutrinos as heavy-lepton neutrinos $\nu_{x}$, all of which experience only the same set of neutral-current interactions.

Our code implements a fixed-mesh-refinement algorithm to simultaneously cover a large spatial region and resolve the compact objects. Specifically, our computational domains consist of seven cuboids centered at an approximate center of mass of the system, and each of the cuboids has $(2 N+1,2 N+1, N+1)$ Cartesian grid points in $(x, y, z)$ directions with the equatorial symmetry imposed at $z=0$. By denoting the region covered by the largest and coarsest domain by $[-L: L] \times[-L: L] \times[0: L]$, the grid spacing of the largest domain is $(\Delta x)_{0}=L / N$. The size and grid spacing of the adjacent domain are halved simultaneously, and thus our smallest and finest domain covers $\left[-L / 2^{6}: L / 2^{6}\right] \times\left[-L / 2^{6}: L / 2^{6}\right] \times\left[0: L / 2^{6}\right]$ with its grid spacing $\Delta x \equiv(\Delta x)_{6}=L /\left(2^{6} N\right)$. Precise values of $L$ and $\Delta x$ are presented in Table II for each model. For the DD2 model, we also perform a low-resolution simulation denoted by DD2-low to check the resolution dependence of our results (see Sec. IIID). 


\section{RESULT}

In this section, we describe the results of neutrino radiation-hydrodynamics simulations of black holeneutron star binary mergers. Throughout this paper, we define the time of merger, $t_{\text {merge }}$, by the time when the baryon rest mass of $0.01 M_{\odot}$ is swallowed by the black hole (strictly speaking, the apparent horizon) following our previous work [3, 10, 11, 85, 86. The time of merger defined in this manner is earlier by up to $1.5 \mathrm{~ms}$ than the time when half of the material is swallowed (see, e.g., Refs. [9, 87]).

\section{A. Merger dynamics and remnant disk}

The inspiral and merger phases are essentially unaffected by the neutrino transport (see Ref. 88 for reviews of purely hydrodynamic simulations). Our initial data are chosen to complete $\sim 3-4$ inspiral orbits taking $\sim 10 \mathrm{~ms}$ before the onset of merger. For the binary parameters adopted in this study, the neutron star is always disrupted by the tidal force of the black hole before the plunge. While most of the disrupted material immediately feeds the black hole, an outer part forms a tidal tail spiraling around the central remnant. The outermost part of the tail is ejected dynamically from the system, and we discuss the mass ejection separately in Sec. IIIC. The irrelevance of neutrinos during this phase is expected and has been found [8, 9], because no heating mechanism raises the neutron-star temperature from the initial value (except for the interaction with the artificial atmosphere, which is found to be dynamically unimportant in our simulations).

Once the tidal tail collides itself and the shock heating sets in, a remnant disk is formed with typical temperature of $\gtrsim 10 \mathrm{MeV}$ and neutrinos begin to emerge. Figures 1 , 2 and 3 show the rest-mass density, temperature, and electron fraction, respectively, in the central region at $10 \mathrm{~ms}$ after the onset of merger. The rest-mass density inside the remnant disk exceeds $10^{12} \mathrm{~g} \mathrm{~cm}^{-3}$ for the models considered in this study. The electron fraction in the dense part is $\lesssim 0.2$ at this time even though the temperature is as high as $10 \mathrm{MeV}$. Figure 3 shows that the electron fraction in the polar region is moderately high, but the mass of such material is very small as the isodensity contours imply.

Remnant disks formed after black hole-neutron star binary mergers show qualitative differences from equilibrium tori commonly adopted as initial conditions of black hole-disk simulations [13, 89 91. The angular momentum profile is close to Keplerian particularly in the innermost region [5, 9], and the disk is geometrically thinner than an equilibrium torus with constant specific angular momentum. We also find that the remnant disk formed from a compact neutron star such as SFHo exhibits an extended region with $Y_{e} \approx 0.2$, which is higher than $Y_{e}=0.1$ commonly adopted as initial conditions of black hole-disk simulations (see also Ref. 92]). These features should have various implications for the postmerger dynamics as we discuss in Sec. V.

\section{B. Neutrino emission}

Figure 4 shows the evolution of the neutrino luminosity for all the models and flavors. All the models dominantly emit electron neutrinos and antineutrinos with the peak luminosity of $\sim 0.5-2 \times 10^{53} \mathrm{erg} \mathrm{s}^{-1}$ for each flavor at $\approx 10 \mathrm{~ms}$ after the onset of merger. Because the selfcollision of the tidal tail occurs only after a single revolution around the black hole (see also Ref. [11), the onset of neutrino emission is delayed by $\approx 10 \mathrm{~ms}$ with respect to the tidal disruption of the neutron star. The emission is dominated by electron/positron captures onto free nucleons (see also below for the discussion of the pair process). The luminosity is higher for $\bar{\nu}_{e}$ by 20\%-30\% than for $\nu_{e}$ as in the case of binary-neutron-star mergers [17, 19, 93 95], because neutrons are more abundant than protons in the accretion disk formed from neutron stars. This hierarchy has also been found in previous studies for the merger of black hole-neutron star binaries [8, 9, 96].

The peak luminosity is higher for the model with a softer equation of state and thus a smaller neutron-star radius such as SFHo. This trend is consistent with the mergers of binary neutron stars [17 19, 63] $]^{3}$ For black hole-neutron star binaries, this dependence of the neutrino luminosity on the neutron-star radius immediately means that the peak luminosity is anticorrelated with the disk mass, because it is established by various previous work that the disk mass is an increasing function of the neutron-star radius for given binary parameters [88]. Figure 5 shows the time evolution of the mass remaining outside the apparent horizon. As expected, the larger the neutron-star radius, the larger the mass outside the apparent horizon. Quantitatively, the values at $10 \mathrm{~ms}$ after the onset of merger agree approximately with those found in our purely hydrodynamic studies performed adopting piecewise polytropic equations of state for comparable neutron-star compactnesses [3] (see also Ref. [97]). This agreement confirms the irrelevance of the neutrino transport in the inspiral and merger phases.

The variation of the neutrino luminosity cannot be ascribed to the spin of the remnant black hole, which is not different substantially among the models. Specifically, the black-hole spins are $0.84-0.86$ at $10 \mathrm{~ms}$ after the onset of merger, which agree with those obtained in our purely hydrodynamic studies [3] (see also Ref. 98]), and the accretion increases them by $0.01-0.02$ in $30 \mathrm{~ms}$. We find that the spin parameter is the largest for SFHo

\footnotetext{
3 This trend seems to be consistent with previous simulations of black hole-neutron star binary mergers 9], whereas it is not explicitly stated.
} 

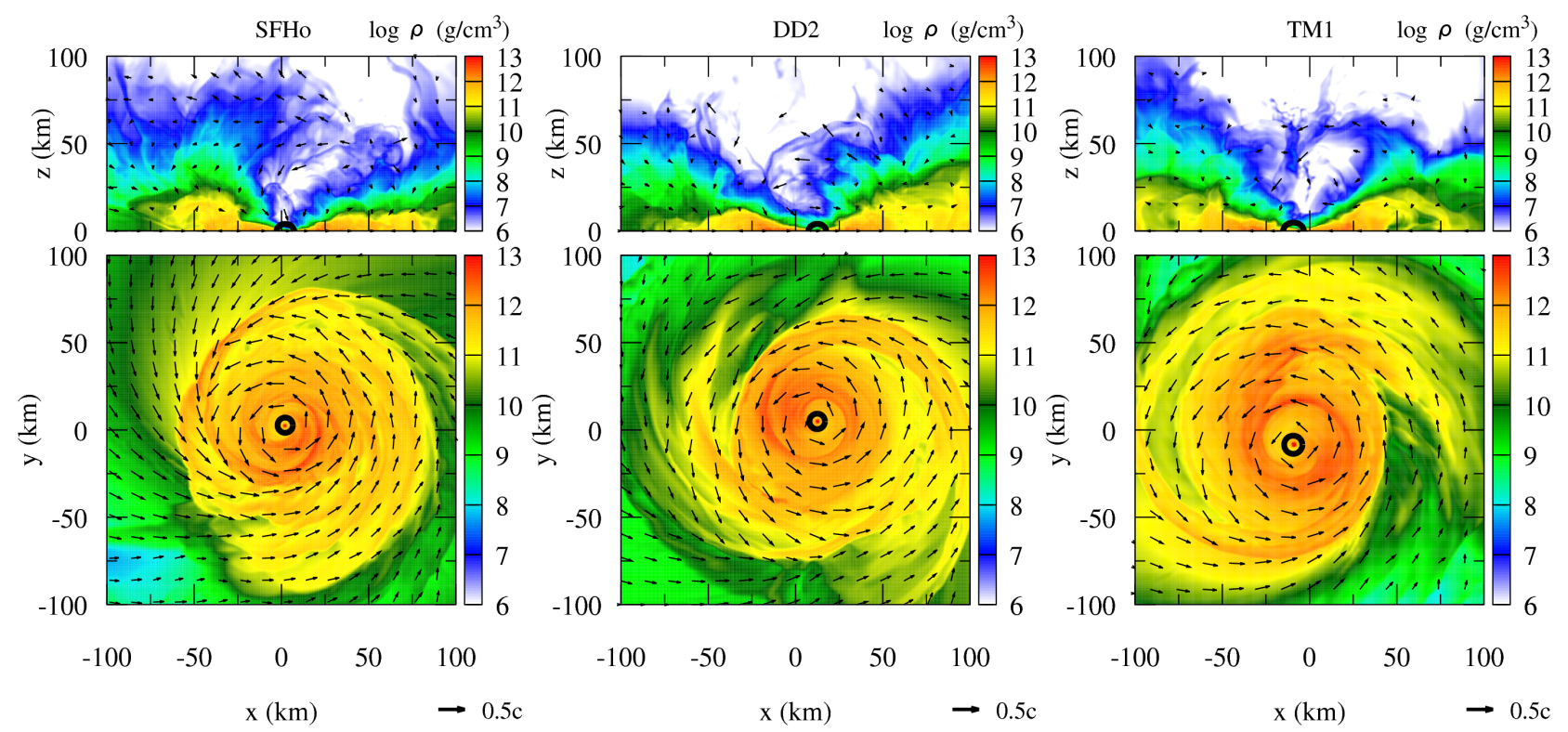

FIG. 1. Rest-mass density profiles in the central region at $10 \mathrm{~ms}$ after the onset of merger, which approximately corresponds to the peak of neutrino luminosity. The black circles show the apparent horizons. The velocity vector $v^{i} \equiv u^{i} / u^{t}$ is overplotted.

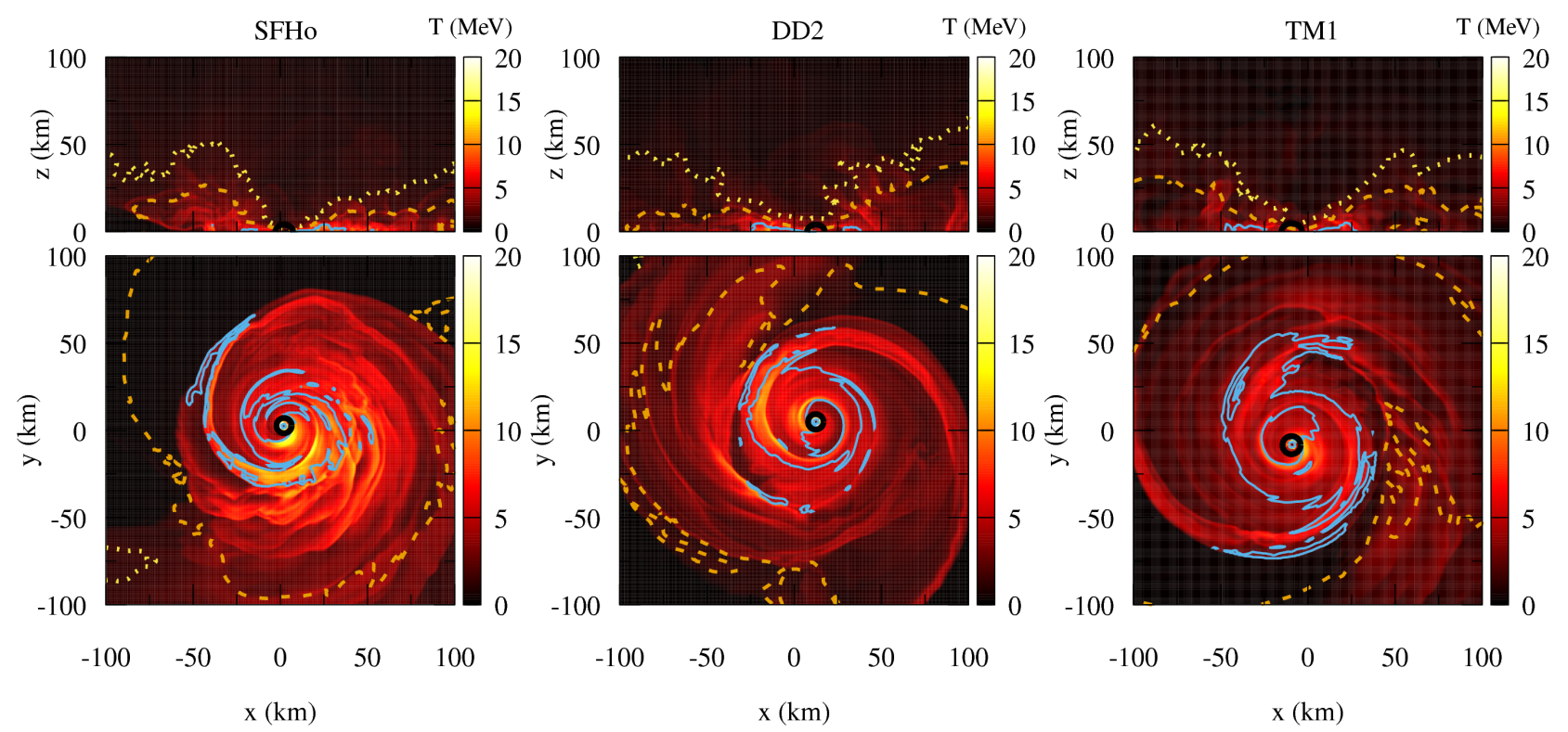

FIG. 2. The same as Fig. 1 but for the temperature. We also show isodensity contours for $\rho=10^{8}$ (yellow dotted), $10^{10}$ (orange dashed), and $10^{12} \mathrm{~g} \mathrm{~cm}^{-3}$ (light blue solid).

and the smallest for TM1, but the difference is very minor.

The high neutrino luminosity for a small neutron-star radius is ascribed to high temperature realized in a compact accretion disk. Figure 6 compares the time evolution of the maximum temperature in the accretion disk and shows that the temperature is higher for smaller neutron stars around $t-t_{\text {merge }} \approx 10 \mathrm{~ms}$, i.e., the peak of the neutrino luminosity. For neutron stars with a small radius such as SFHo, tidal disruption occurs at an orbit very close to the innermost stable circular orbit of the black hole. Accordingly, the self-collision of the tidal tail occurs also near the innermost stable circular orbit. Reflecting high orbital velocity of the close orbit, the energy liberation near the black hole results in the high temperature. Because the emissivity of neutrinos by electron/positron captures is sensitive to the temperature [82, 84], the neutrino luminosity becomes high for soft equations of state and small neutron-star radii.

The rest-mass density may have less impact on the neu- 


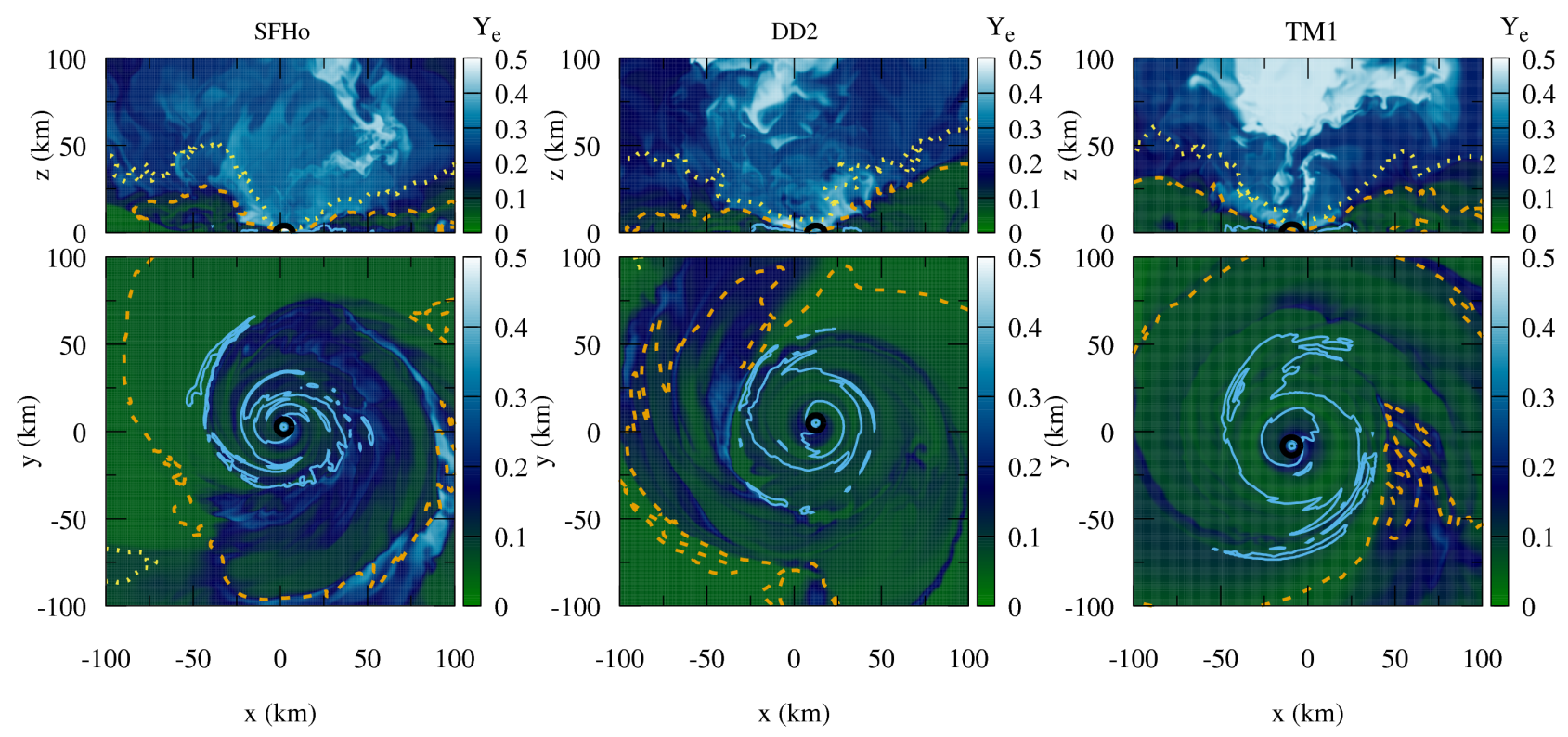

FIG. 3. The same as Fig. 1 but for the electron fraction. We also show isodensity contours for $\rho=10^{8}$ (yellow dotted), $10^{10}$ (orange dashed), and $10^{12} \mathrm{~g} \mathrm{~cm}^{-3}$ (light blue solid). The mass of material with a high electron fraction in the polar region is very small.

trino luminosity. Figure 7 shows the time evolution of the maximum rest-mass density in the accretion disk, which is higher for TM1 than for SFHo on average. The higher rest-mass density for a larger neutron-star radius has already been found in our purely hydrodynamic study as the correlation between the maximum rest-mass density and the disk mass 85, 86. Although the high rest-mass density of the disk for TM1 could be potentially advantageous for neutrino emission, the high temperature of the disk for SFHo plays a dominant role in determining the neutrino luminosity. Note that the high rest-mass density also increases the optical depth and obscures the inner hot portion to some extent, and the values of $Y_{e}$ in the outer part of the disk are increased by neutrino irradiation.

We do not observe strong emission of heavy-lepton neutrinos from the merger remnant of black hole-neutron star binaries. In fact, we are required to multiply the luminosity by a factor of 10 to make the curve for $\nu_{x}$ visible in Fig. 4. This weakness should be compared with moderately strong emission from massive neutron stars formed after binary-neutron-star mergers [17, 19, 95, 99, for which heavy-lepton neutrinos are emitted via pair processes such as the electron-positron pair annihilation. This difference reflects the different temperature of merger remnants. While the massive neutron stars formed after binary-neutron-star mergers are as hot as a few tens of $\mathrm{MeV}$, typical temperature of the accretion disk formed after black hole-neutron star binary mergers is around $10 \mathrm{MeV}$. This is not sufficient to produce a copious amount of heavy-lepton neutrinos via pair processes. Note that the difference of the heavy-lepton neutrino luminosity between SFHo and TM1 is by a factor of 7-8 around the peak, whereas those of the electron neutrino and antineutrino luminosity are at most by a factor of 2. This reflects the steep dependence of the pair emission rate on the temperature.

Figure 8 compares the time evolution of the average neutrino energy among the models. The average energy of neutrinos is not distinctively higher for a softer equation of state, say SFHo, at $t-t_{\text {merge }} \approx 10 \mathrm{~ms}$ for any flavor, unlike the maximum temperature in the accretion disk. After $t-t_{\text {merge }} \approx 20 \mathrm{~ms}$, the average neutrino energy for each flavor settles approximately to constant values common among the models. This suggests that the temperature at the neutrino sphere for a given flavor is similar at the late time irrespective of the neutronstar radius. Specifically, the energy settles to $\approx 8,11$, and $13 \mathrm{MeV}$ for $\nu_{e}, \bar{\nu}_{e}$, and $\nu_{x}$, respectively. Note that, however, the average neutrino energy in this work is estimated as the ratio of the energy emission rate (luminosity) to the number emission rate computed within a gray leakage scheme, and thus only semiquantitative. Precise estimation requires simulations with a multienergy transfer scheme (see also Ref. [21]).

The luminosity gradually decreases in time due to the accretion of hot material by the remnant black hole. From Fig. 4, the luminosity is expected to decrease by an order of magnitude from the peak value in $\approx 100 \mathrm{~ms}$ after the onset of merger, which approximately amounts to the accretion time scale of the remnant due to hydrodynamic processes associated with nonaxisymmetric disk structures [9 11, 87. Specifically, the accretion time scale is estimated to be $\approx 130-150 \mathrm{~ms}$ at $\approx 30 \mathrm{~ms}$ after the onset of merger, being consistent with our previous work when measured at a similar epoch [10, 11, 85, 86]. 

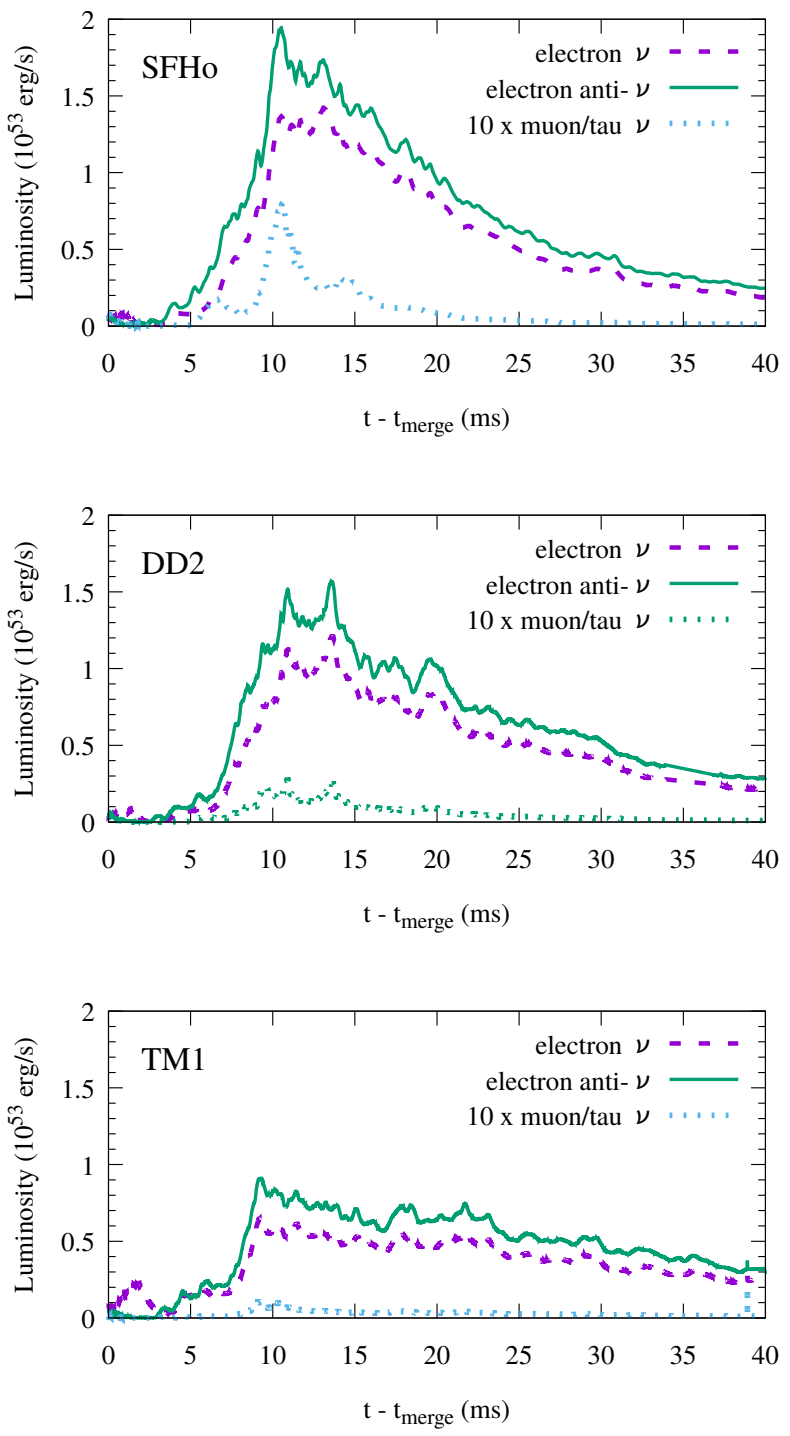

FIG. 4. Neutrino luminosity curves for SFHo (top), DD2 (middle), and TM1 (bottom). The purple-dashed, greensolid, and cyan-dotted curves correspond to the electron neutrino, electron antineutrino, and one of the heavy-lepton neutrinos, respectively. The luminosity curves for the heavylepton neutrinos are multiplied by a factor of 10 to make the plot visible.

The accretion time scale depends only weakly on the grid resolution [10. An effective viscosity may be characterized by $\alpha \sim 0.005-0.01$ in a standard prescription 100 . In reality, however, the temperature of the disk should be increased by viscous heating due to magnetohydrodynamic processes in the accretion phase. If the material is heated efficiently, the neutrino luminosity is enhanced (see, e.g., Refs. [62, 89, 101,103]), and therefore our estimate should be regarded as a lower limit. We would like to revisit this topic in the near future [61, 104, 105.

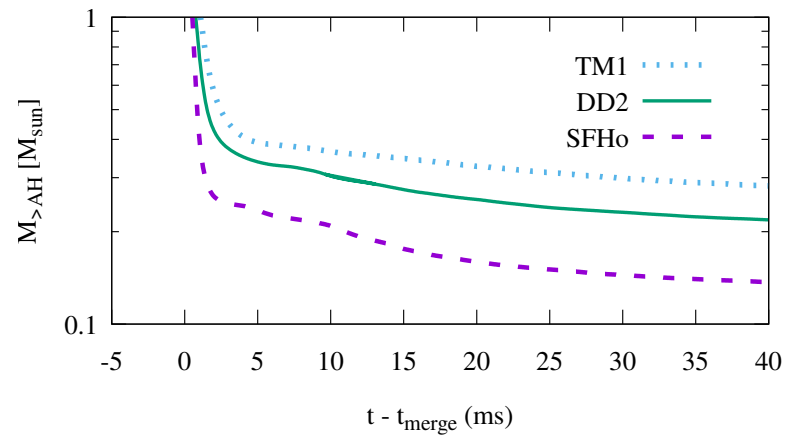

FIG. 5. Time evolution of the mass remaining outside the apparent horizon for SFHo (purple dashed), DD2 (green solid), and TM1 (cyan dotted).

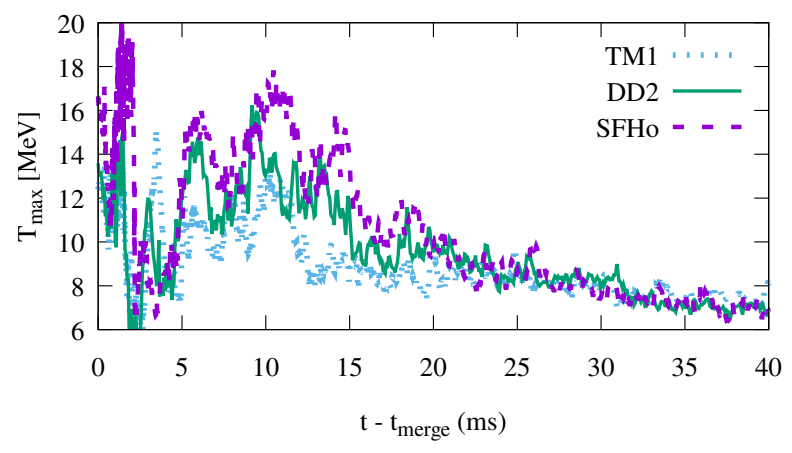

FIG. 6. Time evolution of the maximum temperature in the accretion disk for SFHo (purple dashed), DD2 (green solid), and TM1 (cyan dotted).

\section{Mass ejection}

The outer part of the tidally elongated neutron stars is ejected dynamically at 1-2 ms after the onset of merger [7, 11. Figure 9 shows the rest-mass density, temperature, and electron fraction of the ejecta as well as the bound material on the equatorial plane at $3 \mathrm{~ms}$ after the onset of merger. The amount of dynamical ejecta in the polar region is tiny [7, 11. The ejecta are anisotropic and cold as found in previous work [7, 11. In this work, we further found that the electron fraction is as low as $Y_{e} \lesssim 0.1$ for the most part of the dynamical ejecta, even though the neutrino transport is solved in this work. In the following, we investigate the properties of ejecta quantitatively.

Figure 10 shows the time evolution of the ejecta mass. All the curves in the figure exhibit a steep rise associated with the dynamical mass ejection in a few milliseconds after the onset of merger. The values of the ejecta mass are $\approx 0.01 M_{\odot}, 0.05 M_{\odot}$, and $0.08 M_{\odot}$ for SFHo, DD2, and TM1, respectively, at $10 \mathrm{~ms}$ after the onset of merger. They are compatible with the results of our previous purely hydrodynamic study performed with piece- 


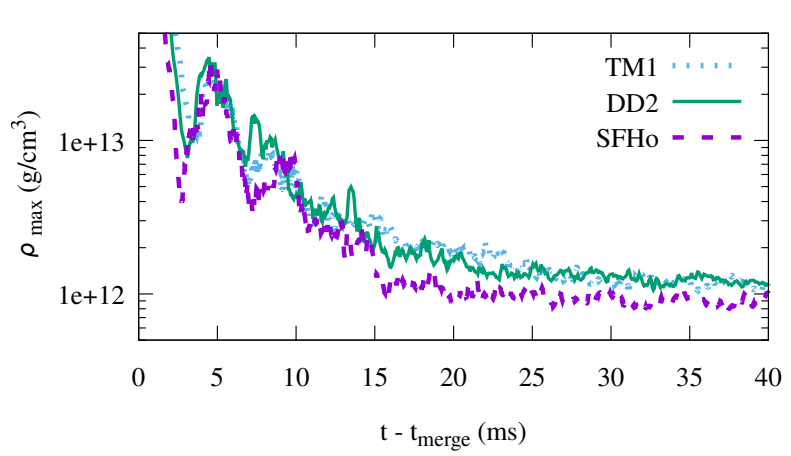

FIG. 7. Time evolution of the maximum rest-mass density in the accretion disk for SFHo (purple dashed), DD2 (green solid), and TM1 (cyan dotted).

wise polytropic equations of state for models with comparable binary parameters [11. We also find that our results are consistent with a fitting formula 4 for dynamical ejecta calibrated against the results of simulations performed employing piecewise polytropes [27. Specifically, the fitting formula predicts $0.009 M_{\odot}, 0.04 M_{\odot}$, and $0.07 M_{\odot}$ for SFHo, DD2, and TM1, respectively. This agreement implies that the fitting formula of Ref. [27] is valid also for finite-temperature equations of state.

Figure 11 shows the time evolution of the ejecta velocity, which is $0.25-0.3 c$ at $10 \mathrm{~ms}$ after the onset of merger. This also agrees with our previous results and the prediction of the fitting formula, $0.25 c$, for the dynamical ejecta [27. However, we see that the ejecta velocity asymptotes to $0.2-0.25 \mathrm{c}$ on a long time scale as they escape from the gravitational binding of the remnant black hole. The decrease by $\sim 20 \%$ is consistent with the estimate in Ref. [11.

The electron fraction of the dynamical ejecta is lower than $Y_{e}=0.1$ for most of the ejecta components. Figure 12 shows the time evolution of the mass-averaged electron fraction, $Y_{e, \mathrm{ej}}$, which is identical to the electron fraction of the entire ejecta. The values fall in the range between 0.05 and 0.07 for all the models. This low electron fraction directly reflects the composition of neutron-star matter in neutrinoless $\beta$-equilibrium at zero temperature. Stated differently, neither the shock heating nor the neutrino irradiation (see Fig. 4) has a significant impact on the dynamical ejecta that leave the central region in a short time scale after the onset of merger.

The averaged electron fraction, $Y_{e, \mathrm{ej}}$, is higher when the ejecta mass is larger for the models considered in this study, and this trend is opposite to that observed in the case of binary neutron stars [17, 19]. Whereas numerical errors associated with the finite resolution prevent us from concluding this correlation to be definitive (see

\footnotetext{
${ }^{4}$ http://www2.yukawa.kyoto-u.ac.jp/ ${ }^{\sim}$ kyohei.kawaguchi/kn_calc/ main.html
}
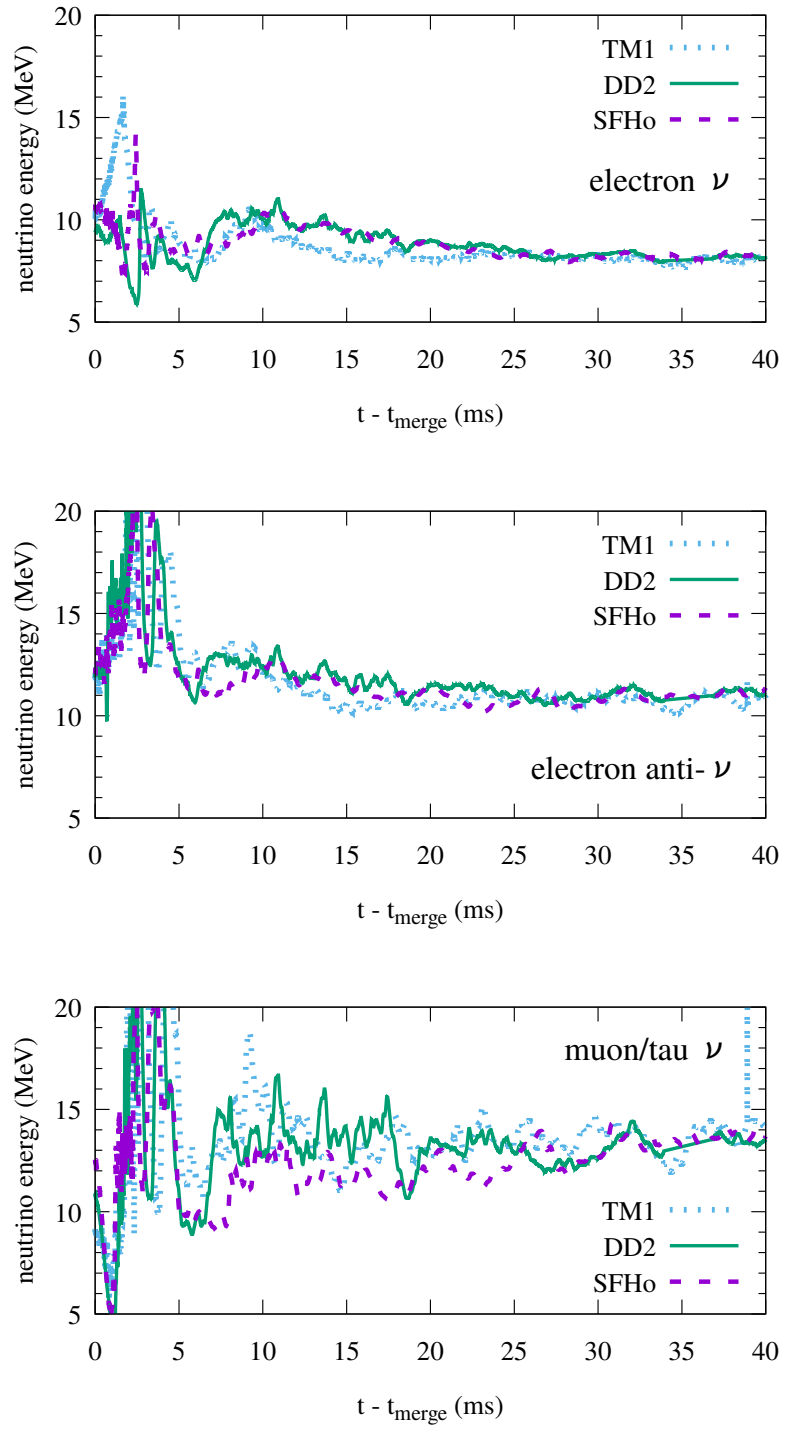

FIG. 8. Time evolution of the average neutrino energy for $\nu_{e}$ (top), $\bar{\nu}_{e}$ (middle), and $\nu_{x}$ (bottom). The purple-dashed, green-solid, and cyan-dotted curves correspond to SFHo, DD2, and TM1, respectively. The average energy of $\bar{\nu}_{e}$ and $\nu_{x}$ approaches $30 \mathrm{MeV}$ at $t-t_{\text {merge }} \approx 3-4 \mathrm{~ms}$, whereas we restrict the range in this figure to focus on long-term behavior.

Sec. III D, we argue below that this can reasonably stem from the correlation between the symmetry energy for nuclear matter and the neutron-star radius [106. When the symmetry energy is higher, the pressure of nuclear matter at and above the saturation density tends to be higher. High pressure at and above the saturation density is empirically found to give a large neutron-star radius [106], and the large radius gives the large ejecta mass for black hole-neutron star binaries. Thus, the symmetry energy can be correlated with the ejecta mass via the tidal effect. At the same time, the material prefers relatively proton-rich conditions characterized by a higher value of $Y_{e}$ when the symmetry energy is higher. Hence, 

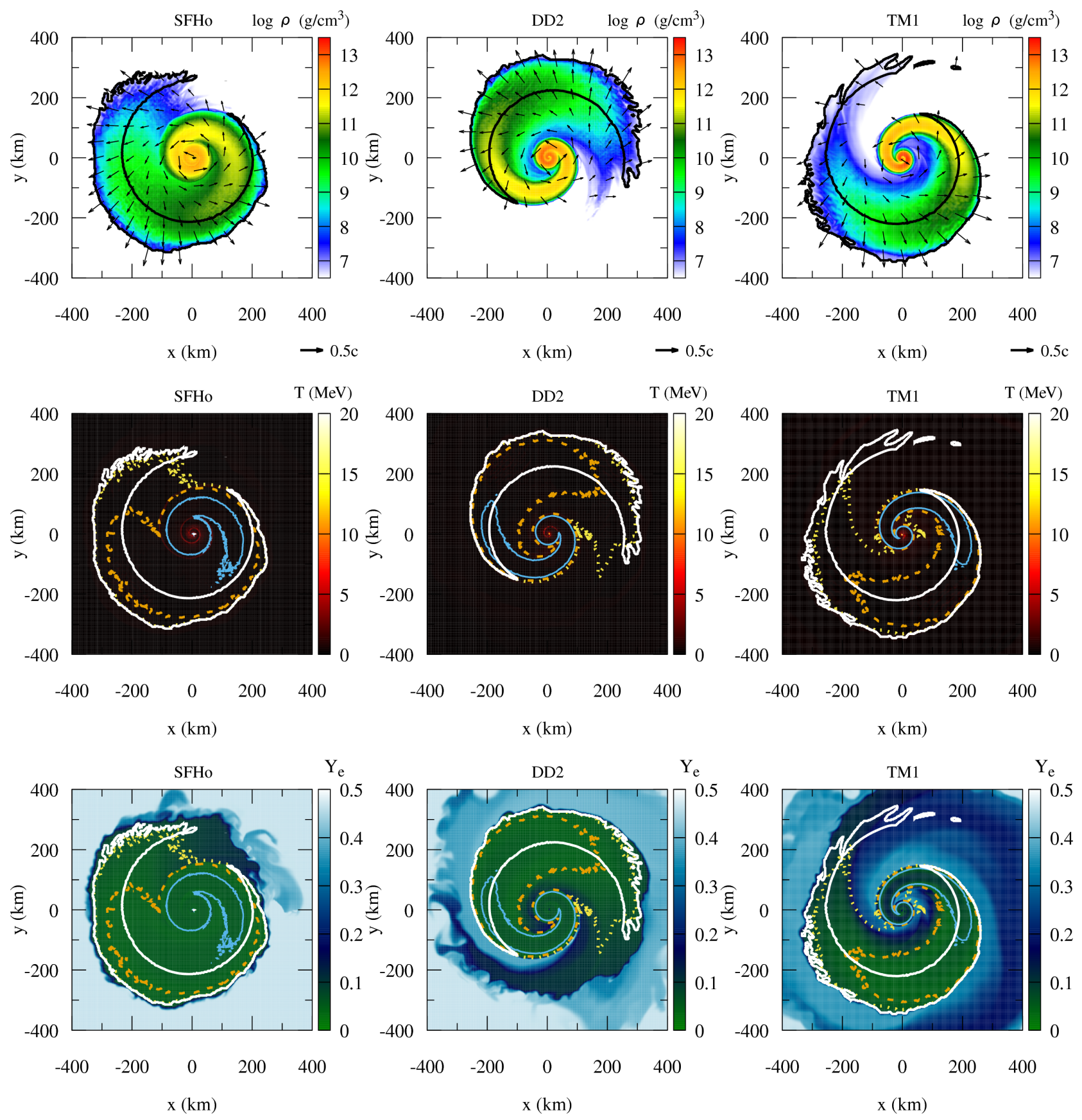

FIG. 9. Rest-mass density (top), temperature (middle), and electron fraction (bottom) profiles on the equatorial plane at $3 \mathrm{~ms}$ after the onset of merger, when the mass and electron fraction of the ejecta approximately settle to asymptotic values. Note the different spatial scale compared to Figs. 1, 2 and 3 . Black (top) and white (middle and bottom) curves indicate the unbound component identified by the conditions $u_{t}<-1$ (shown only for $\rho \geq 10^{6.5} \mathrm{~g} \mathrm{~cm}^{-3}$ to match the top row), which are indistinguishable from $h u_{t}<-1$ on panels presented here [11. The velocity vector $v^{i} \equiv u^{i} / u^{t}$ is overplotted on the rest-mass density profiles. We also show isodensity contours for $\rho=10^{7}$ (yellow dotted), $10^{9}$ (orange dashed), and $10^{11} \mathrm{~g} \mathrm{~cm}{ }^{-3}$ (light blue solid) on the temperature and electron-fraction profiles.

the ejecta mass and averaged electron fraction can be reasonably correlated. Note that the ejecta mass also depends on binary parameters other than the neutron-star radius.

Figures 13 present the mass spectrum of the elec- tron fraction measured at $\approx 10 \mathrm{~ms}$ after the onset of merger. These spectra do not change appreciably in time. The electron fraction peaks sharply around the averaged value, $Y_{e, \text { ej }}$, shown in Fig. 12 The peak shifts slightly towards the high- $Y_{e}$ side as the equation of state becomes 


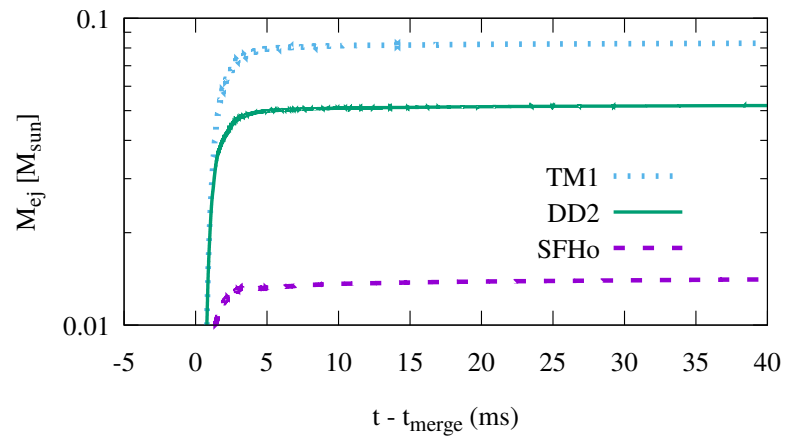

FIG. 10. Time evolution of the ejecta mass identified by the condition $u_{t}<-1$ for SFHo (purple dashed), DD2 (green solid), and TM1 (cyan dotted).

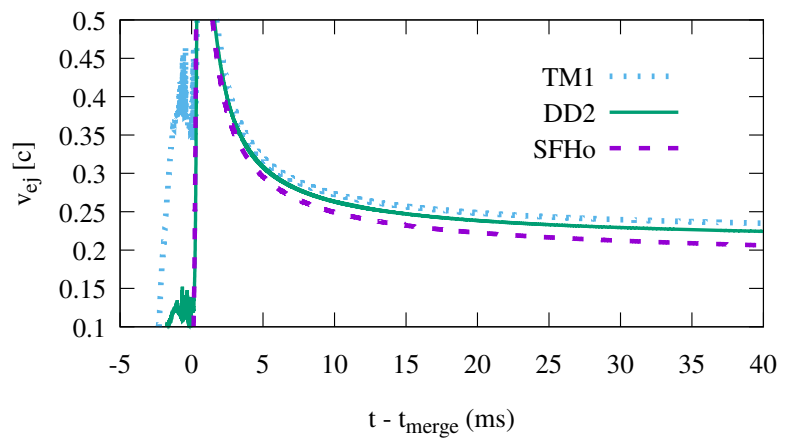

FIG. 11. Time evolution of the ejecta velocity estimated in the same manner as described in Refs. [11, 15] for SFHo (purple dashed), DD2 (green solid), and TM1 (cyan dotted). The values before $t=t_{\text {merge }}$ are numerical artifacts associated with the small ejecta mass. The values right after the onset of merger is $\approx 0.7 c$, but we do not show such transitional values.

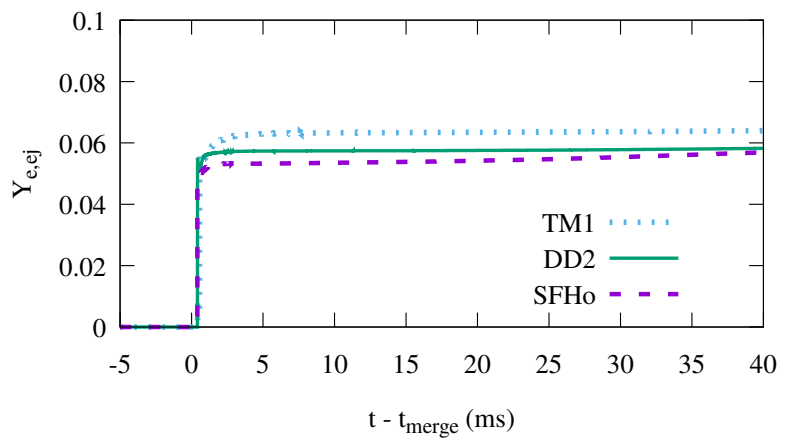

FIG. 12. Time evolution of the mass-averaged electron fraction of the ejecta for SFHo (purple dashed), DD2 (green solid), and TM1 (cyan dotted).

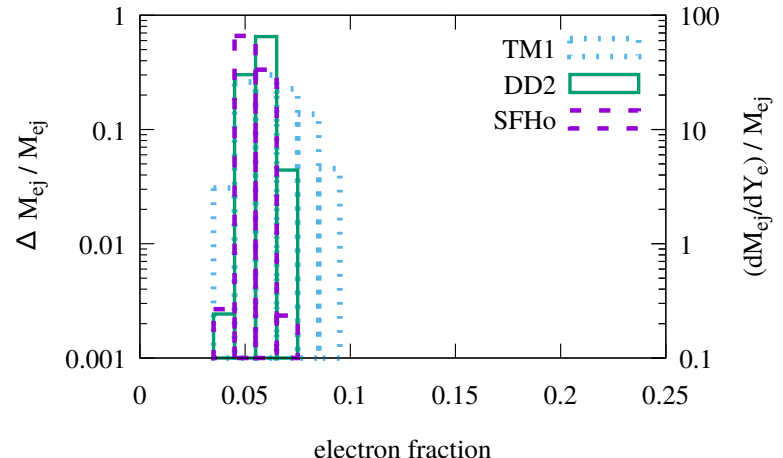

FIG. 13. Distribution of the electron fraction in the unbound material at $\approx 10 \mathrm{~ms}$ after the onset of merger. The left axis shows the fraction of material in the bin of width $\Delta Y_{e}=$ 0.01 , and the right axis shows the differential distribution normalized by the total ejecta mass. The latter is independent of the bin width.

stiff in accordance with Fig. 12. We find that the specific entropy also takes a low value of $\lesssim 10 k_{\mathrm{B}}^{-1}$ irrespective of the models peaking around $5 k_{\mathrm{B}}^{-1}$. This is consistent with the finding that the ejecta do not experience substantial shock heating. The low electron fraction and entropy of the dynamical ejecta found in this study agree with the results of previous simulations performed without neutrino absorption [1, 4, 13, 14.

We observe no substantial mass ejection from the remnant disk due to the neutrino heating, i.e., neutrinodriven wind, in our simulations performed until a few tens milliseconds after the onset of merger. This is evident from the approximately constant values of the ejecta mass at late times (see Fig. 10). The insignificance of the purely neutrino-driven wind has been pointed out in various simulations for the remnant of black hole-neutron star binary mergers with different levels of sophistication [13, 65, 89, 90. Our study confirms this fact by fully general-relativistic radiation-hydrodynamics simulations starting from the inspiral phase for the first time. We caution that our current simulations cannot evaluate the amount of the viscously driven wind and the effect of neutrino irradiation on it, because physical viscosity is not implemented.

\section{Convergence}

We check the convergence of our results by simulating the DD2 model with a low resolution of $\Delta x=400 \mathrm{~m}$, which is coarser by $\approx 50 \%$ than our fiducial run. Figure 14 shows various quantities derived by the high- (discussed so far) and low-resolution simulations. By conservatively assuming first-order convergence, the error in the high-resolution results will be twice the difference between high- and low-resolution ones. While some physical quantities do not converge very well with our current res- 

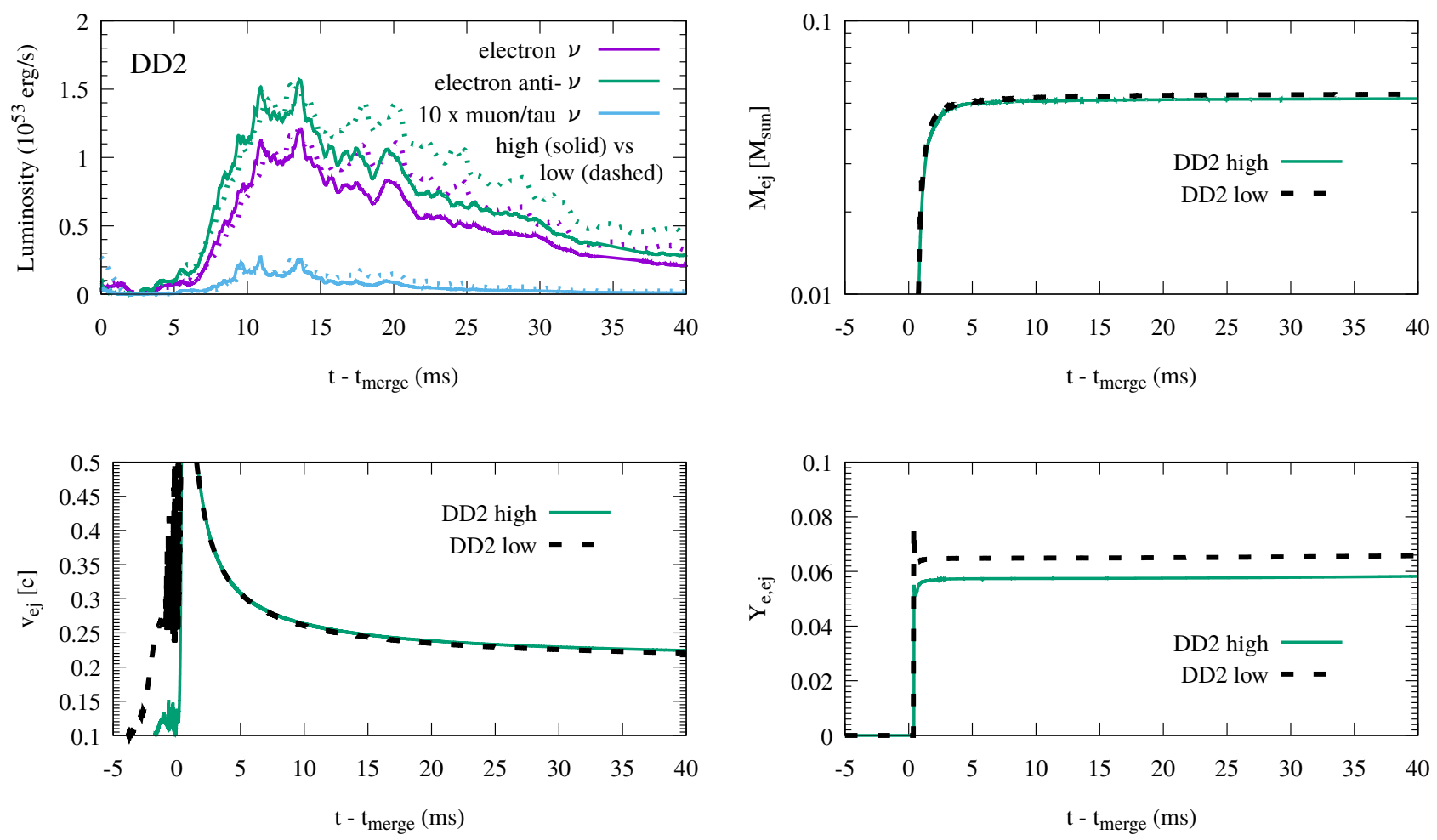

FIG. 14. Comparison of results for the DD2 model between high $(\Delta x=270 \mathrm{~m}$, solid curve) and low ( $\Delta x=400 \mathrm{~m}$, dashed curve) resolutions. The former is the same as those have been shown in other figures. The top-left, top-right, bottom-left, and bottom-right panels show the neutrino luminosity for all the flavors, the ejecta mass, the ejecta velocity, and the averaged electron fraction of the ejecta, respectively. The luminosity curves for the heavy-lepton neutrinos are multiplied by a factor of 10 to make the plot visible in the top-left panel. The velocity right after the onset of merger is out of the vertical range and is not shown in the bottom-left panel as described in the caption of Fig. 11 .

olutions, we may safely conclude that, e.g., the dynamical ejecta from black hole-neutron star binaries have a low electron fraction of $Y_{e} \lesssim 0.1$ as discussed in the following.

The top-left panel of Fig. 14 compares the time evolution of the neutrino luminosity. The peak luminosity agrees within a few $\%$ for the electron neutrino/antineutrino and $10 \%$ for the heavy-lepton neutrino. This agreement implies that our simulations appropriately resolve the process of the disk formation. After the peak, the luminosity is systematically higher by $\sim$ a few tens $\%$ for the low resolution irrespective of the flavors. This difference stems from spurious heating at the low resolution due to enhanced numerical dissipation in the remnant disk. Thus, the late-time luminosity may be overestimated by a factor of up to $\sim 2$ for our fiducial runs under adopted physical inputs. The realistic values should be set by physical viscosity not modeled in this study.

Kinematic properties of the ejecta are approximately convergent. Specifically, Fig. 14 shows that the mass (top right) and velocity (bottom left) agree within 5\% between two resolutions. Thus, the error in the results of the high-resolution run may be less than $10 \%$. Such good convergence is expected for the case in which the ejecta mass is large 11 and may also apply to the other models considered in this study.

The averaged electron fraction of the ejecta shown in the bottom-right panel of Fig. 14 is less convergent than the kinematic properties, although the error does not seem crucial. The difference between the high and low resolutions is $\approx 10 \%$, and the error in the results of the high-resolution run may be as large as $\approx 20 \%$. We observe that the material with high values of $Y_{e}$ is slightly more abundant for the low resolution than for the high resolution, but the mass with $Y_{e} \geq 0.1$ is less than $0.1 \%$ of the ejecta mass and is much smaller than the numerical error. Thus, we may safely conclude that the dynamical ejecta from black hole-neutron star binaries have a low electron fraction of $Y_{e} \lesssim 0.1$.

\section{RESULT WITHOUT NEUTRINO ABSORPTION}

To single out the effect of neutrino transport, we perform a simulation for the DD2 model without incorporating neutrino absorption. The neutrino light curves are compared in Fig. 15. This figure shows that the neutrino 


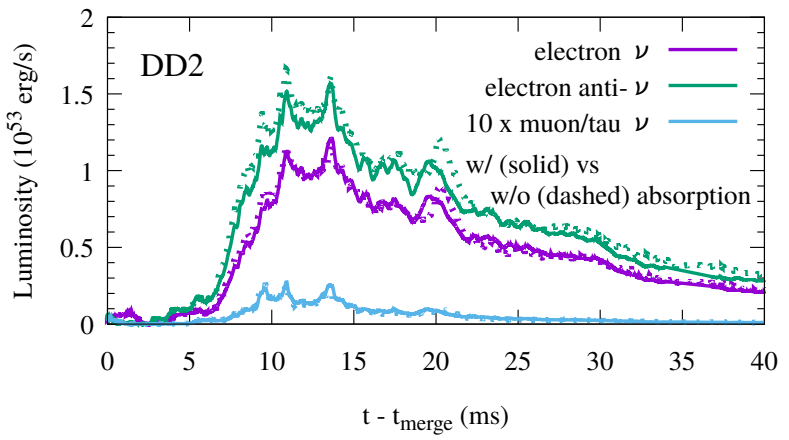

FIG. 15. Neutrino luminosity curves of all the flavors for DD2. The color is the same as Fig. 4. The solid and dashed curves are results for simulations with and without neutrino absorption, respectively, and thus the former is the same as the middle panel of Fig. 4. The luminosity curves for the heavy-lepton neutrinos are multiplied by a factor of 10 to make the plot visible.

emission is not affected substantially by the neutrino absorption onto the material. Note that the optical depth is always taken into account for calculating the neutrino luminosity in the leakage scheme irrespective of the neutrino transport. Close inspection reveals that the luminosity becomes slightly low when the absorption is taken into account as a natural outcome.

The insignificance of the neutrino absorption in the mass ejection from black hole-neutron star binaries is confirmed by comparing the results of simulations with and without neutrino absorption. Figure 16 shows the time evolution of the mass and averaged electron fraction of the ejecta. Neither quantity changes appreciably as a result of the neutrino absorption. The differences are much smaller than the errors associated with the finite resolution discussed in Sec. IIID. This comparison clearly indicates that the purely neutrino-driven wind is negligible and also that the neutrino irradiation does not have a significant impact at least within our approximate transport scheme for this model.

We also perform a simulation without neutrino absorption for the SFHo model up to $t-t_{\text {merge }} \approx 20 \mathrm{~ms}$ and obtain the same conclusion. Because the ejecta mass is smaller for SFHo than for DD2, this comparison gives stronger evidence of the insignificance of the neutrino transport. Quantitatively, the averaged electron fraction, $Y_{e, \mathrm{ej}}$, decreases only by $\lesssim 0.001$.

The ineffectiveness of neutrino absorption can be understood by following analytic arguments. By approximating that the ejecta are composed purely of neutrons, the time scale for neutrino absorption to change the electron fraction of ejecta by (arbitrarily chosen) $\Delta Y_{e, \text { ej }} \approx 0.1$ may be estimated by [see Eq. (3.3) of Ref. [19]

$$
\begin{aligned}
t_{\mathrm{abs}} & \approx \Delta Y_{e, \mathrm{ej}}\left[\frac{1}{4 \pi\left(v_{\mathrm{ej}} t_{\mathrm{exp}}\right)^{2}} \frac{\sigma L_{\nu}}{\left\langle\epsilon_{\nu}\right\rangle}\right]^{-1}, \\
& \approx 100 \mathrm{~ms} \times\left(\frac{t_{\mathrm{exp}}}{10 \mathrm{~ms}}\right)^{2}\left(\frac{L_{\nu}}{10^{53} \mathrm{erg} \mathrm{s}^{-1}}\right)^{-1},
\end{aligned}
$$

where $t_{\exp } \approx t-t_{\text {merge }}$ is the time scale of the expansion of the ejecta, $\sigma \approx 10^{-41} \mathrm{~cm}^{2}$ is the cross section for the capture of neutrinos with average energy of $\left\langle\epsilon_{\nu}\right\rangle \approx 10 \mathrm{MeV}$, and $L_{\nu}$ is the neutrino luminosity, which takes a value of $\approx 10^{53} \mathrm{erg} \mathrm{s}^{-1}$ around the peak of emission, $t_{\exp } \approx 10 \mathrm{~ms}$. The time scale for the appreciable increase of internal energy is longer than this, because the kinetic energy per nucleon, a few tens of $\mathrm{MeV}$, is higher than the average energy of neutrinos. These time scales are always longer by at least an order of magnitude than the expansion time scale of the ejecta, $t_{\text {exp }}$. In reality, $t_{\text {abs }}$ is likely to be longer than this estimate, because neutrinos have to catch up the ejecta, the neutrino luminosity decreases in time, and the ejecta contain a small fraction of protons. Thus, the neutrino absorption should be ineffective, and our simulations confirm this expectation.

We note that our treatment of the neutrino absorption is approximate, while the associated error is expected to be minor. Specifically, we calculate the absorption rate in a local manner assuming that streaming neutrinos obey the Fermi-Dirac distribution with the temperature of the fluid. This prescription is expected to be valid in the optically thick and also intermediate (gray) regions. However, the neutrino temperature is underestimated in the cold and optically thin region, where high temperatures in the emitting region should be appropriate. At the same time, we assign finite chemical potential to streaming neutrinos by requiring that the energy density agrees with the value obtained from the time evolution. Mitigated by this finite chemical potential, the absorption rate is underestimated by typically less than a factor of 2 even in the optically thin region. This amount of the error is unlikely to affect our conclusion that the neutrino absorption is inefficient in dynamical mass ejection from black hole-neutron star binaries. It is also assuring that our results are consistent with those of a postprocess study of neutrino irradiation for black hole-neutron star binaries [55, and the weakness of the purely neutrinodriven wind from the hot remnant disk may be affected only very weakly by this treatment of the temperature. Still, the assumption that neutrinos obey the Fermi-Dirac distribution itself is not strictly valid [107], and multienergy transport simulations are necessary to obtain reliable results (see also Ref. 21]). We leave this task as our future study. 

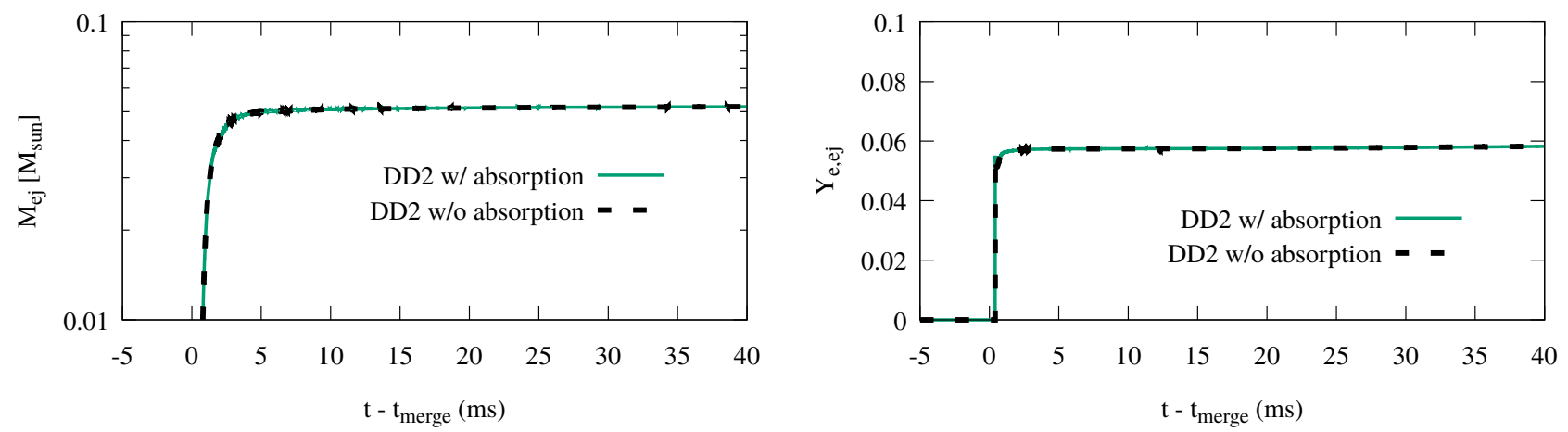

FIG. 16. Time evolution of the mass (left) and averaged electron fraction (right) of ejecta for the DD2 model with (solid) and without (dashed) neutrino absorption.

\section{DISCUSSION}

\section{A. r-process nucleosynthesis and macronova/kilonova}

The very neutron-rich dynamical ejecta from black hole-neutron star binaries are promising as a site of the strong $r$-process nucleosynthesis, which produces the second and third peaks of the abundance pattern. However, it is very unlikely that the dynamical ejecta from black hole-neutron star binaries can produce elements below the second peak, because the ejecta component with the electron fraction appropriate for the first-peak production, say $Y_{e} \gtrsim 0.25$ [50, 55], is very minor even under the neutrino irradiation (see Fig. 13). Furthermore, the purely neutrino-driven wind is negligible even if its electron fraction could be high. Still, the remnants of black hole-neutron star binaries could produce the first-peak elements if the viscously driven wind is strong and only mildly neutron rich 13,64 . Sufficient viscosity may be provided via the magnetorotational instability in the accretion disk [12, 91, and we would like to study the chemical composition of such wind components and associated nucleosynthesis in the near future. Because the electron fraction of the remnant disk is not necessarily as low as $Y_{e}=0.1$, particularly if the neutron star is compact like our SFHo model (see Fig. 3), strong $r$-process nucleosynthesis and lanthanide formation may be suppressed 92.

The dynamical ejecta from black hole-neutron star binaries will give rise to a bright macronova/kilonova due to not only the large mass but also various reasons. Because the lanthanides and actinides must be synthesized enormously for the low electron fraction [108, 109, the opacity should be similar to that of the dynamical ejecta from binary neutron stars except for high- $Y_{e}$ components around the polar direction [17, 19, 50] and will not make a substantial difference in the emission. The luminosity during the early, optically thick phase will be enhanced compared to the spherical ejecta due to the anisotropic geometry, which increases the temperature for a given heating source, and accordingly the color will tend to be blue [7, 11, 26].

Dependence of the heating efficiency on the electron fraction has recently been discussed vigorously [110, 111]. The $r$-process nucleosynthesis under a very low electron fraction produces transuranic elements and even induces fission cycling, and thus the dynamical ejecta from black hole-neutron star binaries should contain many heavy nuclides that experience the fission and/or $\alpha$-decay. Decay heat liberated in these channels is deposited more efficiently to the ejecta than that in the $\beta$-decay, in which the energy can be taken away by escaping neutrinos and $\gamma$ rays. While it is debated whether fissile nuclides remain until the epoch relevant to the macronova/kilonova 110, 111, decay heat of the $\beta$-decay can also be thermalized efficiently in the anisotropic ejecta due to the large optical depth to $\gamma$ rays associated with the high density. Thus, the macronova/kilonova from black hole-neutron star binaries will be brighter than that from binary neutron stars for given values of the ejecta mass and kinetic energy.

\section{B. Short-hard gamma-ray burst}

The key to a successful short-hard gamma-ray burst is the launch of a collimated ultrarelativistic jet. One plausible mechanism for the jet launch is pair annihilation of neutrinos and antineutrinos in the polar region of the black hole [112 114]. In the following, we discuss the implication of the results obtained by our neutrino-radiation-hydrodynamics simulations focusing on this mechanism. Our discussion may not apply to other mechanisms of the jet launch, such as the magnetically driven model [12, 115], whereas neutrino cooling and heating are always essential to determine dynamics and properties of the accretion flow [116.

Our results suggest that a massive accretion disk is not necessarily promising for driving an energetic jet from black hole-neutron star binary mergers. Among the three 
models considered in this work, the peak neutrino luminosity is higher when the equation of state is softer and thus the neutron-star radius is smaller because of the higher temperature in the disk. On another front, the large neutron-star radius is advantageous for producing a massive accretion disk as is well known [3, 85, 86, 97. and is confirmed in this work. Thus, the large disk mass does not necessarily give rise to high neutrino luminosity. Although our simulations do not incorporate viscous heating that will affect late-time behavior, this effect is not likely to modify the peak luminosity substantially, because the temperature achieves an approximately virialized value at the peak of emission due solely to the shock heating. Moreover, the disk mass as an energy source decreases monotonically in time, and the viscosity only accelerates the accretion. Therefore, the viscosity is not likely to produce a later and stronger peak than that seen in this study (but see also Refs. [117, 118]). By considering the quadratic dependence of the neutrino pairannihilation power on the neutrino luminosity [61, 112114, a large disk mass is not necessarily advantageous for launching a high peak-luminosity jet via this process. It should be cautioned that the peak neutrino luminosity may not be directly relevant to the total energy of the jet, because it will be determined by late-time luminosity that can be enhanced by viscosity. The annihilation efficiency can also be enhanced if the viscosity changes the geometry of accretion flows from the disk to a torus.

The accretion disks formed in our models of black holeneutron star binaries are not likely to sustain the jet for a duration longer than $\approx 100 \mathrm{~ms}$. This is significantly shorter than the typical duration of prompt emission for observed short-hard gamma-ray bursts 36. The viscosity neglected in this work will not lengthen the emission time scale, because the accretion time scale will only be decreased by the enhanced angular momentum transport. Although our simulations explore a very limited region of possible parameters, it will not be easy to obtain a sizable disk with the accretion time scale much longer than $100 \mathrm{~ms}$. Thus, it will be challenging for black holeneutron star binaries to explain the duration of typical prompt emission as well as extended emission [119.

The merger remnants of our current simulations are also lacking plausible mechanisms to collimate the jet, while the viscosity is essential to draw a conclusion on this topic. In the case of binary-neutron-star mergers, the dynamical ejecta as well as the disk wind will surround the polar region of the central black hole, and indeed it has been shown that they can collimate a hypothetical jet via hydrodynamic interaction depending on the models 40 44. By contrast, our simulations indicate that neither the dynamical ejecta nor the purely neutrinodriven wind supply substantial material to the polar region in black hole-neutron star binary mergers. This fact does not mean that the hydrodynamic collimation cannot work in black hole-neutron star binaries, because the viscously driven wind together with the neutrino irradiation could be responsible for feeding the polar region.
Note that the authors are not aware of simulations for jet propagation starting from geometrically thin initial disk configurations in this context. We would like to revisit this topic in the near future with viscous-hydrodynamics simulations [104, 105].

\section{SUMMARY}

We performed a series of neutrino-radiationhydrodynamics simulations in full general relativity for black hole-neutron star binary mergers. We adopt an approximate but self-consistent neutrino transport scheme including both emission (cooling) and absorption (heating). The neutron star is modeled by three finitetemperature equations of state (SFHo, DD2, and TM1), whereas we focus only on the cases that the neutron-star mass $\left(1.35 M_{\odot}\right)$, black-hole mass $\left(5.4 M_{\odot}\right)$, and blackhole spin ( 0.75 in terms of the dimensionless parameter and aligned with the orbital angular momentum) are fixed.

We find that the mergers of our black hole-neutron star binary models result in the formation of massive remnant disks with $0.2-0.4 M_{\odot}$ that emit a copious amount of the electron neutrinos and antineutrinos with the peak luminosity $\sim 0.5-2 \times 10^{53} \mathrm{erg} \mathrm{s}^{-1}$ for each flavor. Electron antineutrinos are brighter by $20 \%-30 \%$ than electron neutrinos due to the predominant positron capture on neutrons in the accretion disk. The emission of heavy-lepton neutrinos is quite minor [9], because the temperature in the remnant disk is not high enough for the pair processes to become efficient. The peak luminosity is higher when the neutron-star radius is smaller among the three models considered in this study due to the higher temperature in the accretion disk.

Properties of dynamical ejecta from black hole-neutron star binaries are basically the same as those estimated in previous purely hydrodynamic simulations [11. In this work, we confirm the expectation that the electron fraction of the dynamical ejecta is very low keeping the initial composition of the cold neutron star. We show, for the first time by merger simulations, that the neutrino irradiation does not affect significantly the properties of the dynamical ejecta such as the electron fraction by comparing the results obtained with and without neutrino absorption. The reason is that the dynamical ejecta escape to a distant region too rapidly to be irradiated by neutrinos from the remnant disk. As a result, the extreme neutron richness of the neutron-star material is approximately preserved during the dynamical mass ejection irrespective of the neutrino transport. This fact indicates that dynamical mass ejection from black hole-neutron star binaries can be studied accurately without implementing a detailed neutrino transport scheme unlike that from binary neutron stars [17, 19]. Our results also suggest that the nucleosynthesis outcome will be dominated by heavy $r$-process nuclei around second and third peaks.

We also find that the remnant disks do not appreciably 
launch neutrino-driven winds. This is consistent with previous studies of accretion disks with different levels of sophistication [13, 65, 89, 90. However, it is premature to conclude that the neutrino transport does not play any role in the mass ejection from black hole-neutron star binaries. If magnetic fields and associated viscosity play an important role in ejecting substantial material from the remnant 12, the neutrino interaction can be important for determining the properties of ejecta. We leave such multiphysics simulations as our future task.

\section{ACKNOWLEDGMENTS}

We thank Francois Foucart and Norita Kawanaka for valuable discussions. Numerical simulations are performed on the supercomputer K at AICS (Project No. hp170313), Cray XC30 at CfCA of National Astronomical Observatory of Japan, and XC30 at Yukawa Institute for Theoretical Physics of Kyoto University. This work is supported by Japanese Society for Promotion of Science (JSPS) KAKENHI Grant-in-Aid for Scientific Research (No. JP26400267, No. JP15H00782, No. JP15K05077, No. JP16H02183, No. JP16H06341, No. JP16H06342, No. JP16K17706, No. JP17H01131, No. JP17K05447, No. JP17H06361, and No. JP17H06363) and a post-K computer project (priority issue No. 9) of Japanese Ministry of Education, Culture, Sports, Science and Technology (MEXT).
[1] S. Rosswog, Astrophys. J. 634, 1202 (2005)

[2] E. Rantsiou, S. Kobayashi, P. Laguna, and F. A. Rasio, Astrophys. J. 680, 1326 (2008).

[3] K. Kyutoku, H. Okawa, M. Shibata, and K. Taniguchi, Phys. Rev. D 84, 064018 (2011)

[4] S. Rosswog, T. Piran, and E. Nakar, Mon. Not. R. Astron. Soc. 430, 2585 (2013)

[5] F. Foucart, M. B. Deaton, M. D. Duez, L. E. Kidder, I. MacDonald, C. D. Ott, H. P. Pfeiffer, M. A. Scheel, B. Szilagyi, and S. A. Teukolsky, Phys. Rev. D 87, 084006 (2013)

[6] G. Lovelace, M. D. Duez, F. Foucart, L. E. Kidder, H. P. Pfeiffer, M. A. Scheel, and B. Szilágyi, Classical Quantum Gravity 30, 135004 (2013)

[7] K. Kyutoku, K. Ioka, and M. Shibata, Phys. Rev. D 88, $041503(2013)$

[8] M. B. Deaton, M. D. Duez, F. Foucart, E. O'Connor, C. D. Ott, L. E. Kidder, C. D. Muhlberger, M. A. Scheel, and B. Szilagyi, Astrophys. J. 776, 47 (2013)

[9] F. Foucart, M. B. Deaton, M. D. Duez, E. O'Connor, C. D. Ott, R. Haas, L. E. Kidder, H. P. Pfeiffer, M. A. Scheel, and B. Szilagyi, Phys. Rev. D 90, 024026 (2014)

[10] K. Kawaguchi, K. Kyutoku, H. Nakano, H. Okawa, M. Shibata, and K. Taniguchi, Phys. Rev. D 92, 024014 (2015).

[11] K. Kyutoku, K. Ioka, H. Okawa, M. Shibata, and K. Taniguchi, Phys. Rev. D 92, 044028 (2015).

[12] K. Kiuchi, Y. Sekiguchi, K. Kyutoku, M. Shibata, K. Taniguchi, and T. Wada, Phys. Rev. D 92, 064034 (2015)

[13] O. Just, A. Bauswein, R. A. Pulpillo, S. Goriely, and H.-T. Janka, Mon. Not. R. Astron. Soc. 448, 541 (2015)

[14] F. Foucart, D. Desai, W. Brege, M. D. Duez, D. Kasen, D. A. Hemberger, L. E. Kidder, H. P. Pfeiffer, and M. A. Scheel, Classical Quantum Gravity 34, 044002 (2017).

[15] K. Hotokezaka, K. Kiuchi, K. Kyutoku, H. Okawa, Y.-i. Sekiguchi, M. Shibata, and K. Taniguchi, Phys. Rev. D 87, 024001 (2013).

[16] A. Bauswein, S. Goriely, and H.-T. Janka, Astrophys. J. 773, 78 (2013)
[17] Y. Sekiguchi, K. Kiuchi, K. Kyutoku, and M. Shibata, Phys. Rev. D 91, 064059 (2015)

[18] C. Palenzuela, S. L. Liebling, D. Neilsen, L. Lehner, O. L. Caballero, E. O'Connor, and M. Anderson, Phys. Rev. D 92, 044045 (2015)

[19] Y. Sekiguchi, K. Kiuchi, K. Kyutoku, M. Shibata, and K. Taniguchi, Phys. Rev. D 93, 124046 (2016).

[20] D. Radice, F. Galeazzi, J. Lippuner, L. F. Roberts, C. D. Ott, and L. Rezzolla, Mon. Not. R. Astron. Soc. 460, 3255 (2016)

[21] F. Foucart, E. O'Connor, L. Roberts, L. E. Kidder, H. P. Pfeiffer, and M. A. Scheel, Phys. Rev. D 94, 123016 (2016)

[22] J. M. Lattimer and D. N. Schramm, Astrophys. J. 192, L145 (1974).

[23] L.-X. Li and B. Paczyński, Astrophys. J. 507, L59 (1998)

[24] S. R. Kulkarni, (2005), arXiv:astro-ph/0510256.

[25] B. D. Metzger, G. Martínez-Pinedo, S. Darbha, E. Quataert, A. Arcones, D. Kasen, R. Thomas, P. Nugent, I. V. Panov, and N. T. Zinner, Mon. Not. R. Astron. Soc. 406, 2650 (2010).

[26] M. Tanaka, K. Hotokezaka, K. Kyutoku, S. Wanajo, K. Kiuchi, Y. Sekiguchi, and M. Shibata, Astrophys. J. 780, 31 (2014)

[27] K. Kawaguchi, K. Kyutoku, M. Shibata, and M. Tanaka, Astrophys. J. 825, 52 (2016)

[28] B. P. Abbott, R. Abbott, T. D. Abbott, M. R. Abernathy, F. Acernese, K. Ackley, C. Adams, T. Adams, P. Addesso, R. X. Adhikari, and et al., Phys. Rev. Lett. 116, 061102 (2016)

[29] B. P. Abbott, R. Abbott, T. D. Abbott, M. R. Abernathy, F. Acernese, K. Ackley, C. Adams, T. Adams, P. Addesso, R. X. Adhikari, and et al., Phys. Rev. Lett 116, 241103 (2016)

[30] B. P. Abbott, R. Abbott, T. D. Abbott, F. Acernese, K. Ackley, C. Adams, T. Adams, P. Addesso, R. X. Adhikari, V. B. Adya, and et al., Phys. Rev. Lett. 118, 221101 (2017)

[31] B. P. Abbott, R. Abbott, T. D. Abbott, F. Acernese, K. Ackley, C. Adams, T. Adams, P. Addesso, R. X. 
Adhikari, V. B. Adya, and et al., Phys. Rev. Lett. 119, 141101 (2017)

[32] B. P. Abbott, R. Abbott, T. D. Abbott, F. Acernese, K. Ackley, C. Adams, T. Adams, P. Addesso, R. X. Adhikari, V. B. Adya, and et al., Astrophys. J. 851, L35 (2017)

[33] B. P. Abbott, R. Abbott, T. D. Abbott, M. R. Abernathy, F. Acernese, K. Ackley, C. Adams, T. Adams, P. Addesso, R. X. Adhikari, and et al., Phys. Rev. X 6, 041015 (2016)

[34] W. H. Lee and E. Ramirez-Ruiz, New J. Phys. 9, 17 (2007).

[35] E. Nakar, Phys. Rep. 442, 166 (2007).

[36] E. Berger, Annu. Rev. Astron. Astrophys. 52, 43 (2014)

[37] N. R. Tanvir, A. J. Levan, A. S. Fruchter, J. Hjorth, R. Hounsell, K. Wiersema, and R. L. Tunnicliffe, Nature (London) 500, 547 (2013).

[38] E. Berger, W. Fong, and R. Chornock, Astrophys. J. 774, L23 (2013)

[39] K. Hotokezaka, K. Kyutoku, M. Tanaka, K. Kiuchi, Y. Sekiguchi, M. Shiata, and S. Wanajo, Astrophys. J. 778, L16 (2013)

[40] H. Nagakura, K. Hotokezaka, Y. Sekiguchi, M. Shibata, and K. Ioka, Astrophys. J. 784, L28 (2014)

[41] A. Murguia-Berthier, G. Montes, E. Ramirez-Ruiz, F. De Colle, and W. H. Lee, Astrophys. J. 788, L8 (2014).

[42] P. C. Duffell, E. Quataert, and A. I. MacFadyen, Astrophys. J. 813, 64 (2015).

[43] O. Just, M. Obergaulinger, H.-T. Janka, A. Bauswein, and N. Schwarz, Astrophys. J. 816, L30 (2016)

[44] A. Murguia-Berthier, E. Ramirez-Ruiz, G. Montes, F. De Colle, L. Rezzolla, S. Rosswog, K. Takami, A. Perego, and W. H. Lee, Astrophys. J. 853, L34 (2017).

[45] B. P. Abbott, R. Abbott, T. D. Abbott, F. Acernese, K. Ackley, C. Adams, T. Adams, P. Addesso, R. X. Adhikari, V. B. Adya, and et al., Phys. Rev. Lett. 119, 161101 (2017)

[46] B. P. Abbott, R. Abbott, T. D. Abbott, F. Acernese, K. Ackley, C. Adams, T. Adams, P. Addesso, R. X. Adhikari, V. B. Adya, and et al., Astrophys. J. 848, L12 (2017)

[47] B. P. Abbott, R. Abbott, T. D. Abbott, F. Acernese, K. Ackley, C. Adams, T. Adams, P. Addesso, R. X. Adhikari, V. B. Adya, and et al., Astrophys. J. 848, L13 (2017)

[48] K. P. Mooley et al., (2017), arXiv:1711.11573

[49] J. J. Ruan, M. Nynka, D. Haggard, V. Kalogera, and P. Evans, (2017), arXiv:1712.02809.

[50] S. Wanajo, Y. Sekiguchi, N. Nishimura, K. Kiuchi, K. Kyutoku, and M. Shibata, Astrophys. J. 789, L39 (2014).

[51] C. Freiburghaus, S. Rosswog, and F.-K. Thielemann, Astrophys. J. 525, L121 (1999).

[52] S. Goriely, A. Bauswein, and H.-T. Janka, Astrophys. J. 738, L32 (2011)

[53] O. Korobkin, S. Rosswog, A. Arcones, and C. Winteler, Mon. Not. R. Astron. Soc. 426, 1940 (2012)

[54] S. Goriely, A. Bauswein, O. Just, E. Pllumbi, and H.-T. Janka, Mon. Not. R. Astron. Soc. 452, 3894 (2015)

[55] L. F. Roberts, J. Lippuner, M. D. Duez, J. A. Faber, F. Foucart, J. C. Lomberdi, Jr., S. Ning, C. D. Ott, and M. Ponce, Mon. Not. R. Astron. Soc. 464, 3907 (2017).
[56] C. Sneden, J. J. Cowan, and R. Gallino, Annu. Rev. Astron. Astrophys. 46, 241 (2008)

[57] C. Siqueira Mello, V. Hill, B. Barbuy, M. Spite, F. Spite, T. C. Beers, E. Caffau, P. Bonifacio, R. Cayrel, P. François, H. Schatz, and S. Wanajo, Astron. Astrophys. 565, A93 (2014)

[58] L. Dessart, C. D. Ott, A. Burrows, S. Rosswog, and E. Livne, Astrophys. J. 690, 1681 (2009)

[59] A. Perego, S. Rosswog, R. Cabezón, O. Korobkin, R. Käppeli, A. Arcones, and M. Liebendörfer, Mon. Not. R. Astron. Soc. 443, 3134 (2014).

[60] D. Martin, A. Perego, A. Arcones, F.-K. Thielemann, O. Korobkin, and S. Rosswog, Astrophys. J. 813, 2 (2015)

[61] S. Fujibayashi, Y. Sekiguchi, K. Kiuchi, and M. Shibata, Astrophys. J. 846, 114 (2017)

[62] B. D. Metzger and R. Fernández, Mon. Not. R. Astron. Soc. 441, 3444 (2014)

[63] F. Foucart, R. Haas, M. D. Duez, E. O'Connor, C. D. Ott, L. Roberts, L. E. Kidder, J. Lippuner, H. P. Pfeiffer, and M. A. Scheel, Phys. Rev. D 93, 044019 (2016).

[64] M.-R. Wu, R. Fernández, G. Martínez-Pinedo, and B. D. Metzger, Mon. Not. R. Astron. Soc. 463, 2323 (2016)

[65] F. Foucart, E. O'Connor, L. Roberts, M. D. Duez, R. Haas, L. E. Kidder, C. D. Ott, H. P. Pfeiffer, M. A. Scheel, and B. Szilagyi, Phys. Rev. D 91, 124021 (2015).

[66] A. W. Steiner, M. Hempel, and T. Fischer, Astrophys. J. 774, 17 (2013)

[67] S. Banik, M. Hempel, and D. Bandyopadhyay, Astrophys. J. Suppl. 214, 22 (2014)

[68] M. Hempel, T. Fischer, J. Schaffner-Bielich, and M. Liebendörfer, Astrophys. J. 748, 70 (2012).

[69] K. Hebeler, J. M. Lattimer, C. J. Pethick, and A. Schwenk, Phys. Rev. Lett. 105, 161102 (2010)

[70] I. Tews, J. M. Lattimer, A. Ohnishi, and E. E. Kolomeitsev, Astrophys. J. 848, 105 (2017).

[71] P. Demorest, T. Pennucci, S. Ransom, M. Roberts, and J. Hessels, Nature (London) 467, 1081 (2010).

[72] J. Antoniadis et al., Science 340, 1233232 (2013)

[73] K. Kyutoku, M. Shibata, and K. Taniguchi, Phys. Rev. D 79, 124018 (2009)

[74] L. Kreidberg, C. D. Bailyn, W. M. Farr, and V. Kalogera, Astrophys. J. 757, 36 (2012).

[75] M. Shibata and T. Nakamura, Phys. Rev. D 52, 5428 (1995)

[76] T. W. Baumgarte and S. L. Shapiro, Phys. Rev. D 59, 024007 (1998)

[77] M. Campanelli, C. O. Lousto, P. Marronetti, and Y. Zlochower, Phys. Rev. Lett. 96, 111101 (2006)

[78] J. G. Baker, J. Centrella, D.-I. Choi, M. Koppitz, and J. van Meter, Phys. Rev. Lett. 96, 111102 (2006).

[79] P. Marronetti, W. Tichy, B. Brügmann, J. A. González, and U. Sperhake, Phys. Rev. D 77, 064010 (2008)

[80] K. S. Thorne, Mon. Not. R. Astron. Soc. 194, 439 (1981)

[81] M. Shibata, K. Kiuchi, Y. Sekiguchi, and Y. Suwa, Prog. Theor. Phys. 125, 1255 (2011)

[82] Y. Sekiguchi, Prog. Theor. Phys. 124, 331 (2010).

[83] Y. Sekiguchi and M. Shibata, Astrophys. J. 737, 6 (2011)

[84] Y. Sekiguchi, K. Kiuchi, K. Kyutoku, and M. Shibata, Prog. Theor. Exp. Phys. 2012, 01A304 (2012).

[85] K. Kyutoku, M. Shibata, and K. Taniguchi, Phys. Rev. 
D 82, 044049 (2010).

[86] K. Kyutoku, M. Shibata, and K. Taniguchi, Phys. Rev. D 84, 049902(E) (2011)

[87] F. Foucart, M. D. Duez, L. E. Kidder, and S. A. Teukolsky, Phys. Rev. D 83, 024005 (2011)

[88] M. Shibata and K. Taniguchi, Living Rev. Relativity 14, 6 (2011).

[89] R. Fernández and B. D. Metzger, Mon. Not. R. Astron. Soc. 435, 502 (2013)

[90] R. Fernández, D. Kasen, B. D. Metzger, and E. Quataert, Mon. Not. R. Astron. Soc. 446, 750 (2015)

[91] D. M. Siegel and B. D. Metzger, Phys. Rev. Lett. 119, $231102(2017)$

[92] R. Fernández, F. Foucart, D. Kasen, J. Lippuner, D. Desai, and L. F. Roberts, Class. Quantum Grav. 34, 154001 (2017)

[93] M. Ruffert, H.-T. Janka, K. Takahashi, and G. Schäfer, Astron. Astrophys. 319, 122 (1997).

[94] S. Rosswog and M. Liebendörfer, Mon. Not. R. Astron. Soc. 342, 673 (2003)

[95] Y. Sekiguchi, K. Kiuchi, K. Kyutoku, and M. Shibata, Phys. Rev. Lett. 107, 051102 (2011)

[96] H.-T. Janka, T. Eberl, M. Ruffert, and C. L. Fryer, Astrophys. J. 527, L39 (1999).

[97] F. Foucart, Phys. Rev. D 86, 124007 (2012).

[98] F. Pannarale, Phys. Rev. D 88, 104025 (2013).

[99] Y. Sekiguchi, K. Kiuchi, K. Kyutoku, and M. Shibata, Phys. Rev. Lett. 107, 211101 (2011)

[100] S. Kato, J. Fukue, and S. Mineshige, Black-hole accretion disks - towards a new paradigm - (Kyoto University Press, 2008).

[101] R. Popham, S. E. Woosley, and C. Fryer, Astrophys. J. $\mathbf{5 1 8}, 356$ (1999)

[102] K. Kohri and S. Mineshige, Astrophys. J. 577, 311 (2002).
[103] M. Shibata, Y.-i. Sekiguchi, and R. Takahashi, Prog. Theor. Phys. 118, 257 (2007)

[104] M. Shibata, K. Kiuchi, and Y.-i. Sekiguchi, Phys. Rev. D 95, 083005 (2017)

[105] M. Shibata and K. Kiuchi, Phys. Rev. D 95, 123003 (2017)

[106] J. M. Lattimer and M. Prakash, Astrophys. J. 550, 426 (2001)

[107] S. Richers, D. Kasen, E. O’Connor, R. Fernández, and C. D. Ott, Astrophys. J. 813, 38 (2015).

[108] M. Tanaka and K. Hotokezaka, Astrophys. J. 775, 113 (2013).

[109] D. Kasen, N. R. Badnell, and J. Barnes, Astrophys. J. 774, 25 (2013)

[110] K. Hotokezaka, S. Wanajo, M. Tanaka, A. Banba, Y. Terado, and T. Piran, Mon. Not. R. Astron. Soc. 459, 35 (2016)

[111] J. Barnes, D. Kasen, M.-R. Wu, and G. MartínezPinedo, Astrophys. J. 829, 110 (2016)

[112] J. Goodman, A. Dar, and S. Nussinov, Astrophys. J. 314, L7 (1987)

[113] D. Eichler, M. Livio, T. Piran, and D. N. Schramm, Nature (London) 340, 126 (1989).

[114] R. Mochkovitch, M. Hernanz, J. Isern, and X. Martin, Nature (London) 361, 236 (1993).

[115] V. Paschalidis, M. Ruiz, and S. L. Shapiro, Astrophys. J. 806, L14 (2015)

[116] N. Kawanaka, T. Piran, and J. H. Krolik, Astrophys. J. 766, 31 (2013)

[117] W. H. Lee, E. Ramirez-Ruiz, and D. Page, Astrophys. J. 632, 421 (2005)

[118] S. Setiawan, M. Ruffert, and H.-T. Janka, Astron. Astrophys. 458, 553 (2006)

[119] Y. Kagawa, D. Yonetoku, T. Sawano, A. Toyanago, T. Nakamura, K. Takahashi, K. Kashiyama, and K. Ioka, Astrophys. J. 811, 4 (2015). 